\title{
Results from coalbed methane drilling in Winn Parish, Louisiana
}

By Paul C. Hackley ${ }^{1}$, Peter D. Warwick ${ }^{1}$, F. Clayton Breland, Jr. ${ }^{2}$, Troy E. Richard $^{3}$, and Kirk Ross ${ }^{4}$

This report is preliminary and has not been reviewed for conformity with U.S. Geological Survey editorial standards and stratigraphic nomenclature. Any use of trade, product, or firm names is for descriptive purposes only, and does not imply endorsement by the U.S. Geological Survey.

\section{Open-File Report 2007-1061}

${ }^{1}$ U.S. Geological Survey, Reston, VA 20192

${ }^{2}$ Louisiana Geological Survey, Basin Research Energy Section, Baton Rouge, LA 70803

${ }^{3}$ Hilcorp Energy Company

${ }^{4}$ Vintage Petroleum, Inc. 


\section{U.S. Department of the Interior \\ Dirk A. Kempthorne, Secretary}

\section{U.S. Geological Survey \\ Mark D. Myers, Director}

U.S. Geological Survey, Reston, Virginia: 2007

For product and ordering information:

World Wide Web: http://www.usgs.gov/pubprod

Telephone: 1-888-ASK-USGS

For more information on the USGS-the Federal source for science about the Earth, its natural and living resources, natural hazards, and the environment:

World Wide Web: http://www.usgs.gov

Telephone: 1-888-ASK-USGS

Suggested citation:

Hackley, P.C., Warwick, P.D., Breland, F.C., Jr., Richard, T.E., and Ross, K., 2007, Results from coalbed methane drilling in Winn Parish, Louisiana: U.S. Geological Survey Open-File Report 2007-1061, 45 p. http://www.pubs.usgs.gov/of/2007/1061.

Any use of trade, product, or firm names is for descriptive purposes only and does not imply endorsement by the U.S. Government.

Although this report is in the public domain, permission must be secured from the individual copyright owners to reproduce any copyrighted material contained within this report. 


\section{TABLE OF CONTENTS}

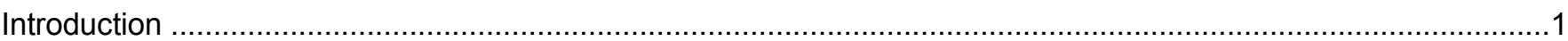

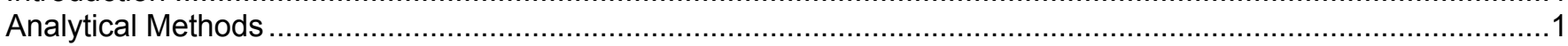

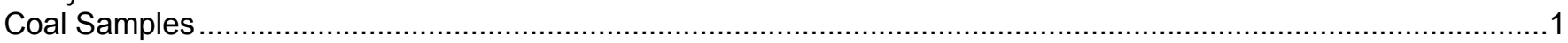

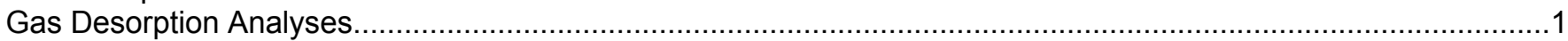

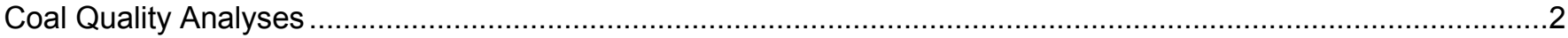

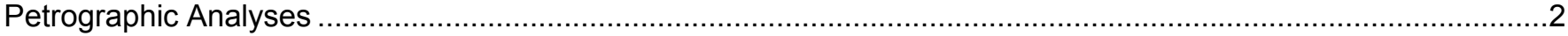

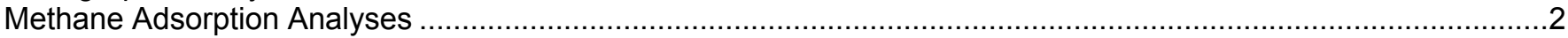

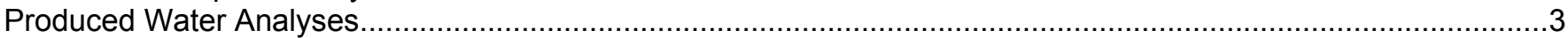

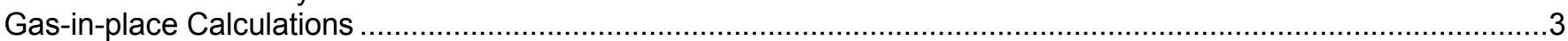

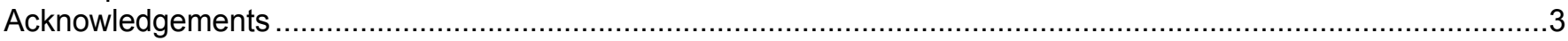

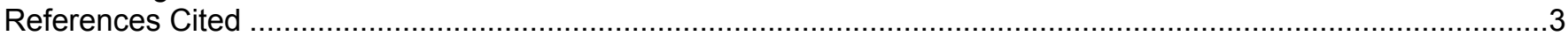

\section{LIST OF FIGURES}

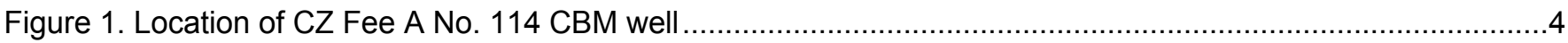

Figure 2. E-logs and interpreted stratigraphy of CZ Fee A No. 114 CBM well. ...............................................

Figure 3. Ash yield (as-received) versus bulk density, CZ Fee A No. 114 CBM well samples ..............................6

Figure 4. Raw gas content versus bulk density, CZ Fee A No. 114 CBM well samples ......................................

Figure 5. A. Lost gas estimation, and B. Cumulative gas volume for CZ Fee A No. 114 CBM well, sample $1 \ldots \ldots \ldots . .8$

Figure 6. A. Lost gas estimation, and B. Cumulative gas volume for CZ Fee A No. 114 CBM well, sample 2 2.........9

Figure 7. A. Lost gas estimation, and B. Cumulative gas volume for CZ Fee A No. 114 CBM well, sample 3 ........10

Figure 8. A. Lost gas estimation, and B. Cumulative gas volume for CZ Fee A No. 114 CBM well, sample $9 \ldots \ldots \ldots . .11$

Figure 9. A. Lost gas estimation, and B. Cumulative gas volume for CZ Fee A No. 114 CBM well, sample 10 ......112

Figure 10. A. Lost gas estimation, and B. Cumulative gas volume for CZ Fee A No. 114 CBM well, sample $11 \ldots . .13$

Figure 11. A. Lost gas estimation, and B. Cumulative gas volume for CZ Fee A No. 114 CBM well, sample 12 ....14

Figure 12. A. Lost gas estimation, and B. Cumulative gas volume for CZ Fee A No. 114 CBM well, sample 13 ....15

Figure 13. Methane adsorption isotherm, CZ Fee A No. 114 CBM well, sample 4, raw basis.............................16

Figure 14. Methane adsorption isotherm, CZ Fee A No. 114 CBM well, sample 4, dry basis .............................16

Figure 15. Methane adsorption isotherm, CZ Fee A No. 114 CBM well, sample 4, dry, ash-free basis .................11

Figure 16. Methane adsorption isotherm, CZ Fee A No. 114 CBM well, sample 7, raw basis............................17

Figure 17. Methane adsorption isotherm, CZ Fee A No. 114 CBM well, sample 7, dry basis ............................18

Figure 18. Methane adsorption isotherm, CZ Fee A No. 114 CBM well, sample 7, dry, ash-free basis .................18

Figure 19. Methane adsorption isotherm, CZ Fee A No. 114 CBM well, sample 11, raw-basis ..........................19

Figure 20. Methane adsorption isotherm, CZ Fee A No. 114 CBM well, sample 11, dry basis ...........................19

Figure 21. Methane adsorption isotherm, CZ Fee A No. 114 CBM well, sample 11, dry, ash-free basis ...............20

Figure 22. Methane adsorption isotherms and measured gas content, CZ Fee A No. 114 CBM well, as-analyzed basis

\section{LIST OF TABLES}

Table 1. General information for CZ Fee A No. 114 CBM well, Winn Parish, LA ............................................22

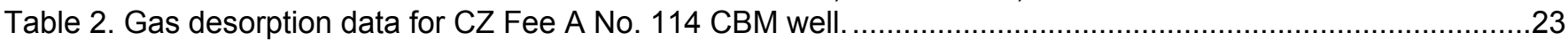

Table 3. Coal quality data for CZ Fee A No. 114 CBM well, sample 4 (2,679.0-2,681.0 ft)............................24

Table 4. Coal quality data for CZ Fee A No. 114 CBM well, sample 7 (2,731.0-2,732.0 ft)............................25

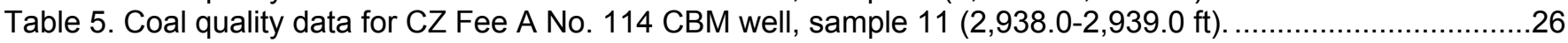

Table 6. Petrographic data for CZ Fee A No. 114 CBM well, sample $4(2,679.0-2,681.0 \mathrm{ft}) . \ldots \ldots \ldots \ldots \ldots \ldots \ldots \ldots \ldots \ldots . .27$

Table 7. Petrographic data for CZ Fee A No. 114 CBM well, sample $7(2,731.0-2,732.0 \mathrm{ft}) \ldots \ldots \ldots \ldots \ldots \ldots \ldots \ldots \ldots \ldots . .28$

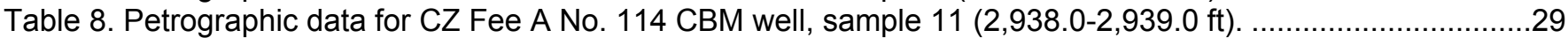

Table 9. Vitrinite reflectance data for CZ Fee A No. 114 CBM well, sample $4(2,679.0-2,681.0 \mathrm{ft}) \ldots \ldots \ldots \ldots \ldots \ldots \ldots . . .30$

Table 10. Vitrinite reflectance data for CZ Fee A No. 114 CBM well, sample 7 (2,731.0-2,732.0 ft)...................31

Table 11. Vitrinite reflectance data for CZ Fee A No. 114 CBM well, sample $11(2,938.0-2,939.0 \mathrm{ft}) \ldots \ldots \ldots \ldots \ldots \ldots \ldots . . .32$

Table 12. Methane adsorption isotherm analytical data (as-analyzed basis) for CZ Fee A No. 114 CBM well, sample 4 . 
Table 13. Methane adsorption isotherm analytical data (dry basis) for CZ Fee A No. 114 CBM well, sample 4 .....34 Table 14. Methane adsorption isotherm analytical data (dry, ash-free basis) for CZ Fee A No. 114 CBM well, sample 4

Table 15. Methane adsorption isotherm analytical data (as-analyzed basis) for CZ Fee A No. 114 CBM well, sample 7 ....

Table 16. Methane adsorption isotherm analytical data (dry basis) for CZ Fee A No. 114 CBM well, sample 7 .....37 Table 17. Methane adsorption isotherm analytical data (dry, ash-free basis) for CZ Fee A No. 114 CBM well, sample 7

Table 18. Methane adsorption isotherm analytical data (as-analyzed basis) for CZ Fee A No. 114 CBM well, sample 11

Table 19. Methane adsorption isotherm analytical data (dry basis) for CZ Fee A No. 114 CBM well, sample 11 ...40 Table 20. Methane adsorption isotherm analytical data (dry, ash-free basis) for CZ Fee A No. 114 CBM well, sample 11

Table 21. Produced water data for CZ Fee A No. 114 well CBM well, February 2004

Table 22. Produced water data for CZ Fee A No. 114 well CBM well, March 2004.

Table 23. Gas-in-place (GIP) calculations for CZ Fee A No. 114 CBM well

\section{APPENDIX}

Permission to publish coalbed methane analytical data from operator 


\title{
Results from coalbed methane drilling in Winn Parish, Louisiana
}

\author{
By Paul C. Hackley, Peter D. Warwick, F. Clayton Breland, Jr., Troy E. Richard,
} and Kirk Ross

\section{INTRODUCTION}

A coalbed methane (CBM) well in Winn

Parish, Louisiana, named CZ Fee A No.

114, was drilled by Vintage Petroleum, Inc., in January 2004. The CZ Fee A No. 114

CBM well was drilled to a total depth of $3,114 \mathrm{ft}$ and perforated at 2,730-2,734 ft in a Wilcox Group (Paleocene-Eocene) coal bed. Analytical data from the drilling project have been released by Vintage Petroleum, Inc., and by the current well operator, Hilcorp Energy Corporation (see Appendix) to the Louisiana Geological Survey (LGS) and the U.S. Geological Survey (USGS) for publication. General information about the CZ Fee A No. 114 CBM well is compiled in Table 1, and analytical data from the well are included in following sections.

The CZ Fee A No. 114 well is located in eastern Winn Parish, approximately $30 \mathrm{mi}$ east of where Wilcox Group strata crop out on the Sabine Uplift (fig. 1). In the CZ Fee A No. 114 well, lower Wilcox Paleocene coal beds targeted for CBM production occur at depths of 2,600-3,000 ft (fig. 2). Average monthly gas production for the reporting period August 1, 2004, through May 1, 2005, was 450 thousand cubic feet (Mcf) (Louisiana Department of Natural Resources, 2005).

\section{ANALYTICAL METHODS}

Coal and water samples obtained from the CZ Fee A No. 114 well were analyzed by contractors to Vintage Petroleum, Inc. Analytical methods used included canister gas desorption (Diamond and Levine, 1981;
Diamond and others, 1986), proximate, heating value, and sulfur content analyses, organic petrographic analysis, and vitrinite reflectance (American Society for Testing of Materials, 2005), adsorption isotherm analysis (Mavor and others, 1990), and produced water quality analysis (Nelson, 2003).

\section{COAL SAMPLES}

Thirteen coal samples were analyzed from three cored intervals in the Wilcox (Table 2). Samples 1 to 5 were recovered from core interval $1(2,673.0$ to $2,683.0 \mathrm{ft})$, samples 6 to 9 were recovered from core interval $2(2,730.0$ to $2,734.0 \mathrm{ft})$, and samples 10 to 13 were recovered from core interval 3 (2,937.0 to $2,941.0 \mathrm{ft}$ ) (fig. 2).

\section{GAS DESORPTION ANALYSES}

Results of canister desorption analyses of the 13 coal core samples are shown in Table 2. Ash yield versus bulk density and raw gas content versus bulk density are plotted in figs. 3 and 4, respectively. Gas content increases with depth and is inversely related to ash content and bulk density. Lost gas content was determined by the U.S. Bureau of Mines direct method (Diamond and Levine, 1981); plots for lost gas estimation and cumulative gas volume are shown for 8 samples in figs. 5 through 12. Total gas content was in the range 6.04 to 158.90 standard cubic feet per ton (scf/ton) (raw basis), or 91.88 to 213.49 scf/ton on a dry, ash-free basis. Residual gas measurements (Diamond and others, 
1986) were performed for four samples

(Table 2) and determined to range 1.93 to $5.56 \mathrm{scf} /$ ton. Moisture content determined on the desorbed samples ranges from 8.45 to 19.88 weight percent (wt.\%) on an asreceived basis (as-rec'd) (Table 2); ash content determined on the desorbed samples ranges from 6.30 to 85.16 wt. \% (as-rec'd) (Table 2).

\section{COAL QUALITY ANALYSES}

Proximate analyses, calorific value, and total sulfur content were determined for three coal samples (samples 4, 7, and 11), and are reported in Tables 3 through 5 . Moisture content ranges from 12.13 to 18.65 wt.\% (as-rec'd). Proximate moisture values are similar to those reported by a different laboratory as part of the gas desorption analyses (1 to $2 \mathrm{wt}$ \% $\%$ absolute differences between the two sets of analyses; compare as-received moisture reported in Tables 3 through 5 with Table 2). Ash yield ranges from 6.29 to 47.41 wt.\% (as-rec'd). Ash yield is similar ( $1 \mathrm{wt} . \%$ absolute differences) to that reported for the gas desorption analyses except for sample 4 where the reported ash yield for the proximate analysis is $10 \mathrm{wt} \%$ greater (absolute) than that reported in the gas desorption analysis (compare as-received ash yield in Table 3 with Table 2). Calorific value ranges from 10,046 to 10,904 British thermal units per pound (Btu/lb) on a moist, mineral-matter free basis and rank according to ASTM D 388 is subbituminous $B-A$. Sulfur content ranges from 0.56 to 1.26 wt. \% (as-rec'd).

\section{PETROGRAPHIC ANALYSES}

\section{Maceral Analyses}

The results of petrographic analyses for samples 4, 7, and 11 are shown in Tables 6 through 8. Samples contain 3.5 to 51.4 volume percent (vol.\%) mineral matter, 41.6 to 60.5 vol. $\%$ vitrinite, 1.8 to 32.2 vol. $\%$ inertinite, and 2.9 to 5.2 vol.\% liptinite (maceral composition on mineral-containing basis). Samples are dominated by detrovitrinite (47.8 to 69.8 vol.\%; mineralfree basis), with subordinate telovitrinite. The high inertinite sample 11 (33.3 vol.\% inertinite, mineral-free basis) comes from the deepest coal core (cored interval 3; $2,937.0$ to $2,941.0 \mathrm{ft}$ ). The high value for inertinite content in sample 11 is much higher than the range of inertinite values (2 to $15 \mathrm{vol} . \%$, mineral-free) reported by Hackley and Warwick (2005) for subsurface Wilcox coals located approximately $60 \mathrm{mi}$ to the north in Ouachita Parish, Louisiana.

\section{Vitrinite Reflectance Analyses}

The results of measurements of the random reflectance of vitrinite for samples 4 , 7 , and 11 are reported in Tables 9 through 11. Values range between 0.34 and $39 \%$ reflectance, indicating lignite to subbituminous $\mathrm{C}$ rank. Rank according to the reflectance of vitrinite is slightly lower than calculated by the Parr Formula (based on ASTM D 388). Reported values for the standard deviation of measured random reflectance are 0.05 to 0.075 , indicating high levels of variability in the measured vitrinite populations.

\section{METHANE ADSORPTION ANALYSES}

Methane adsorption isotherm analyses were performed on samples 4, 7, and 11 . Analytical data are included in Tables 12 to 20 and isotherm plots are shown in figs. 13 through 21. Comparison of desorbed gas content with methane adsorption isotherms indicate that the samples range from undersaturated to slightly oversaturated at hydrostatic reservoir pressure (fig. 22).

Reservoir pressures for the cored intervals were estimated by multiplying depth by 0.433 pounds per square inch per foot (psi/ft) (normal hydrostatic gradient; Schlumberger Limited, 2005). Equilibrium moisture values determined for the three samples as part of the isotherm analyses are 2 to $3.5 \mathrm{wt}$ \% greater (absolute basis) than as-received moisture reported as part 
of the desorption analyses, and 4 to 4.5 wt.\% greater than as-received proximate moisture. As-received ash yield determined as part of the isotherm analyses is within 2 $w t . \%$ of ash yield reported for the proximate analyses.

\section{PRODUCED WATER ANALYSES}

Produced waters were collected in February and March, 2004. Water quality data are tabulated in Tables 21 and 22. Total dissolved solids ranged from 82,800 to $84,600$ milligrams per liter ( $\mathrm{mg} / \mathrm{l}) ; \mathrm{pH}$ ranged from 5.85 to 6.58 .

\section{GAS-IN-PLACE CALCULATIONS}

Calculated gas-in-place (GIP) for the 3 cored intervals ranges from 144,515 to 208,479 Mcf for 160 acre centers (Table 23). Total GIP is 547,680 Mcf on 160 acre centers. The number of bulk density values (35) and their reported magnitude in Table 23 is inconsistent with the number of bulk density values (13) reported for gas desorption analyses (Table 2) because cored intervals were sectioned into $0.5 \mathrm{ft}$ sections and bulk density was estimated using the e-log. Ash fraction in Table 23 is calculated from the estimated bulk density; average gas content and moisture values used in the GIP calculations are from the gas desorption analyses (Table 2).

\section{ACKNOWLEDGEMENTS}

The USGS and LGS thank Vintage Petroleum, Inc., and Hilcorp Energy Corporation for sharing data on coalbed methane exploration in Winn Parish, LA. TerraTek Inc. and its contractors conducted the coal analyses; Baker Petrolite conducted the water analyses. Technical reviews by Leslie Ruppert and Alex Karlsen of USGS improved the quality and clarity of this report.

\section{REFERENCES CITED}

American Society for Testing and Materials (ASTM), 2005, Annual Book of ASTM Standards, Petroleum Products, Lubricants, and Fossil Fuels, Gaseous Fuels; Coal and Coke, Section 5, Volume 05.06: Philadelphia, PA, American Society for Testing and Materials, $675 \mathrm{pp}$.

Diamond, W.P., LaScola, J.C., and Hyman, D.M., 1986, Results of direct method determination of the gas content of U.S. coalbeds: U.S. Bureau of Mines Information Circular 9067, 95 p.

Diamond, W.P., and J.R. Levine, 1981, Direct method determination of the gas content of coal: procedures and results: U.S. Bureau of Mines Report of Investigations 8515, $36 \mathrm{p}$.

Hackley, P.C., and Warwick, P.D., 2005, Organic petrography of coals from a coalbed methane test well, Ouachita Parish, Louisiana: U.S. Geological Survey Open-File Report 2005-1134, 22 p., http://pubs.usgs.gov/of/2005/1134/.

Louisiana Department of Natural Resources, 2005, SONRIS: Louisiana Department of Natural Resources website and database at http://sonriswww.dnr.state.la.us/www root/sonris portal 1.htm (Accessed August, 2005).

Mavor, M.J., Owen, L.B., and Pratt, T.J., 1990, Measurement and evaluation of isotherm data: Proceedings of the $65^{\text {th }}$ Annual Technical Conference and Exhibition of the Society of Petroleum Engineers, SPE 20728, New Orleans, Louisiana, p. 157-170.

Nelson, P., 2003, Index to EPA Test Methods: U.S. Environmental Protection Agency Region 1 Library Publication, 85 p., http://www.epa.gov/region01/info/testmet hods/pdfs/testmeth.pdf.

Schlumberger Limited, 2005, Oilfield Glossary: Schlumberger Limited website at http://www.glossary.oilfield.slb.com/ (Accessed October, 2005). 


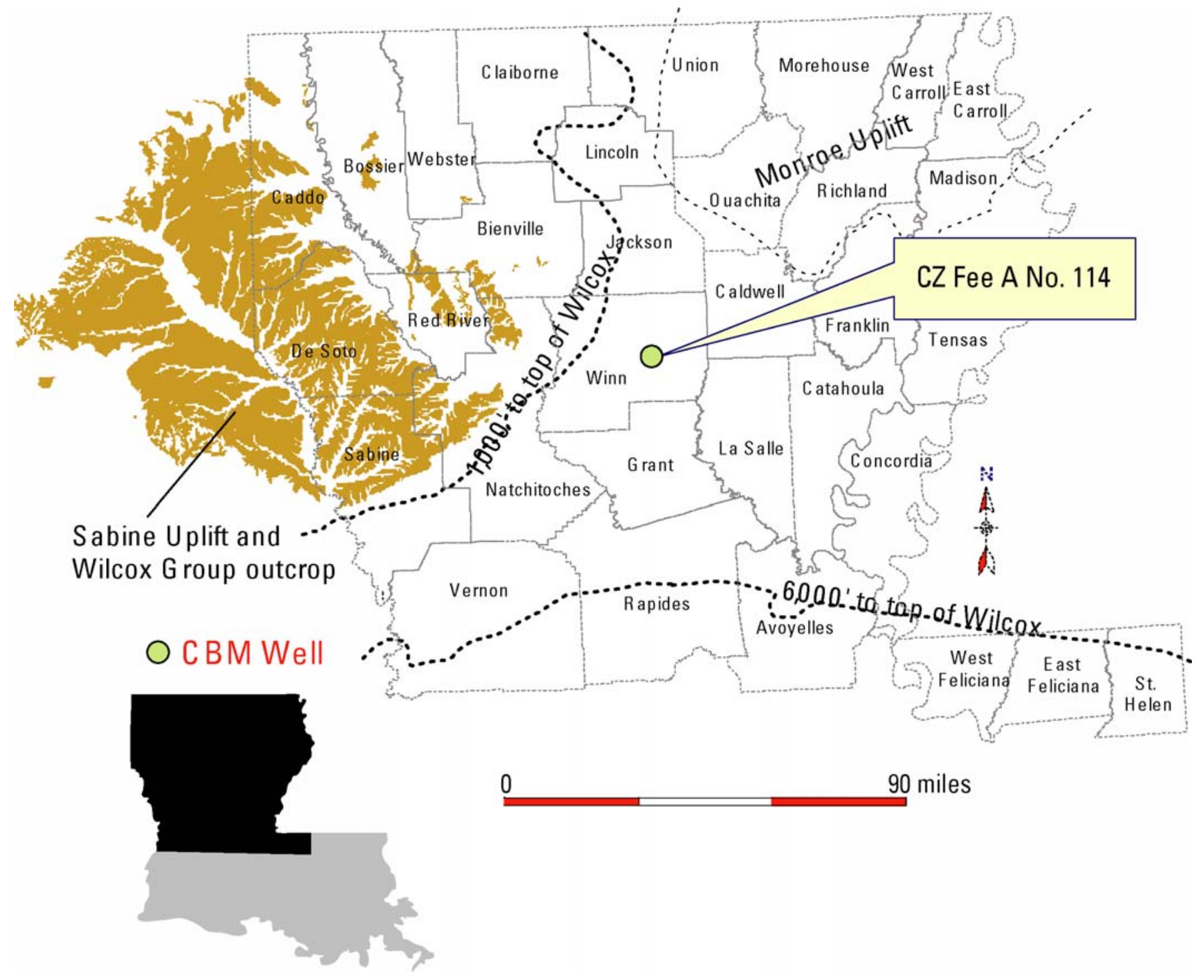

Figure 1. Location of CZ Fee A No. 114 CBM well in Winn County, Louisiana. Heavy dashed line indicates depth to the top of the Wilcox Group. Light dashed line indicates outline of the Monroe Uplift. 


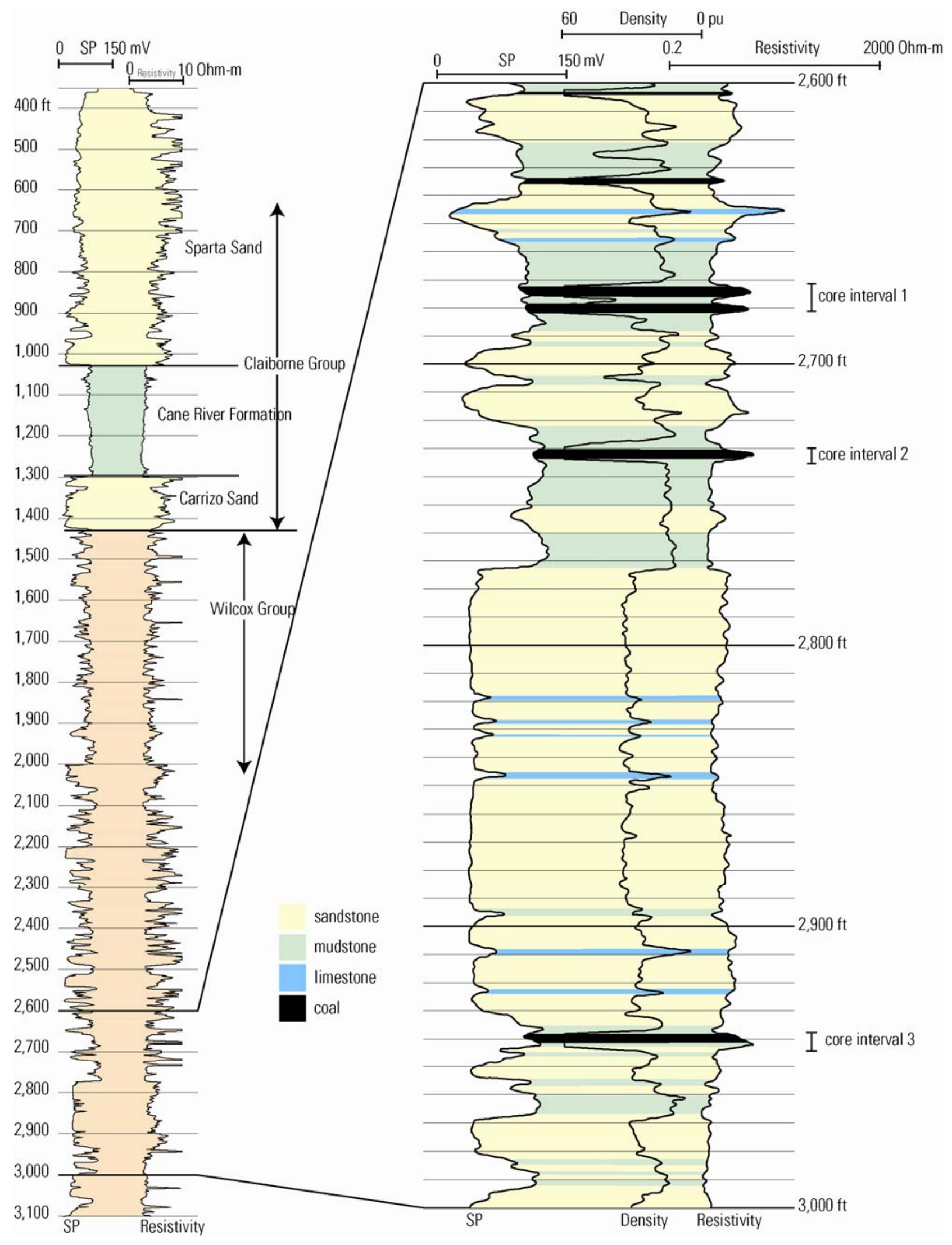

Figure 2. E-logs and interpreted stratigraphy of CZ Fee A No. 114 CBM well. 


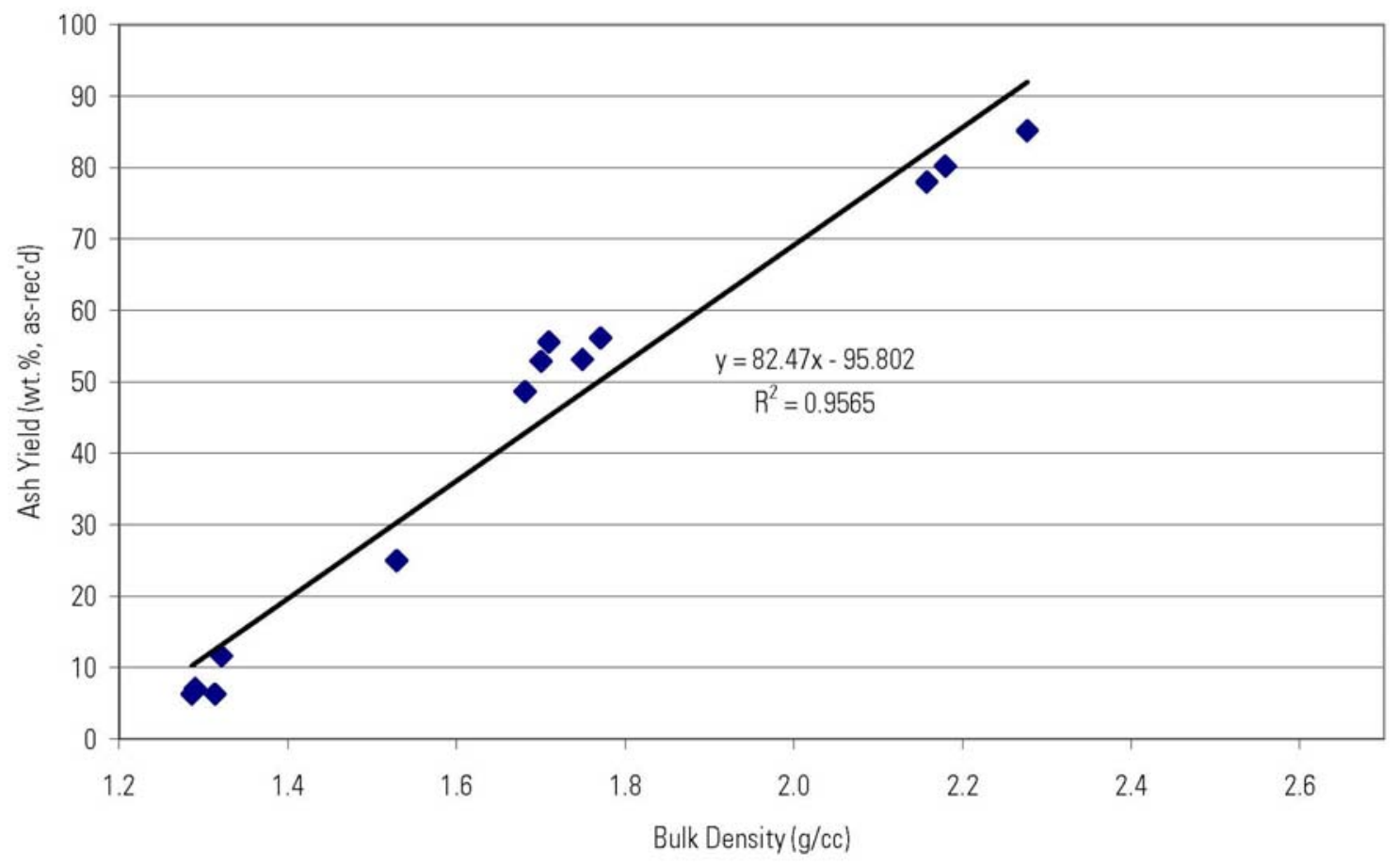

Figure 3. Ash yield (as-received) versus bulk density, CZ Fee A No. 114 CBM well samples. Abbreviations: $\%$ = percent; as-rec'd = as-received; g/cc = grams per cubic centimeter. 


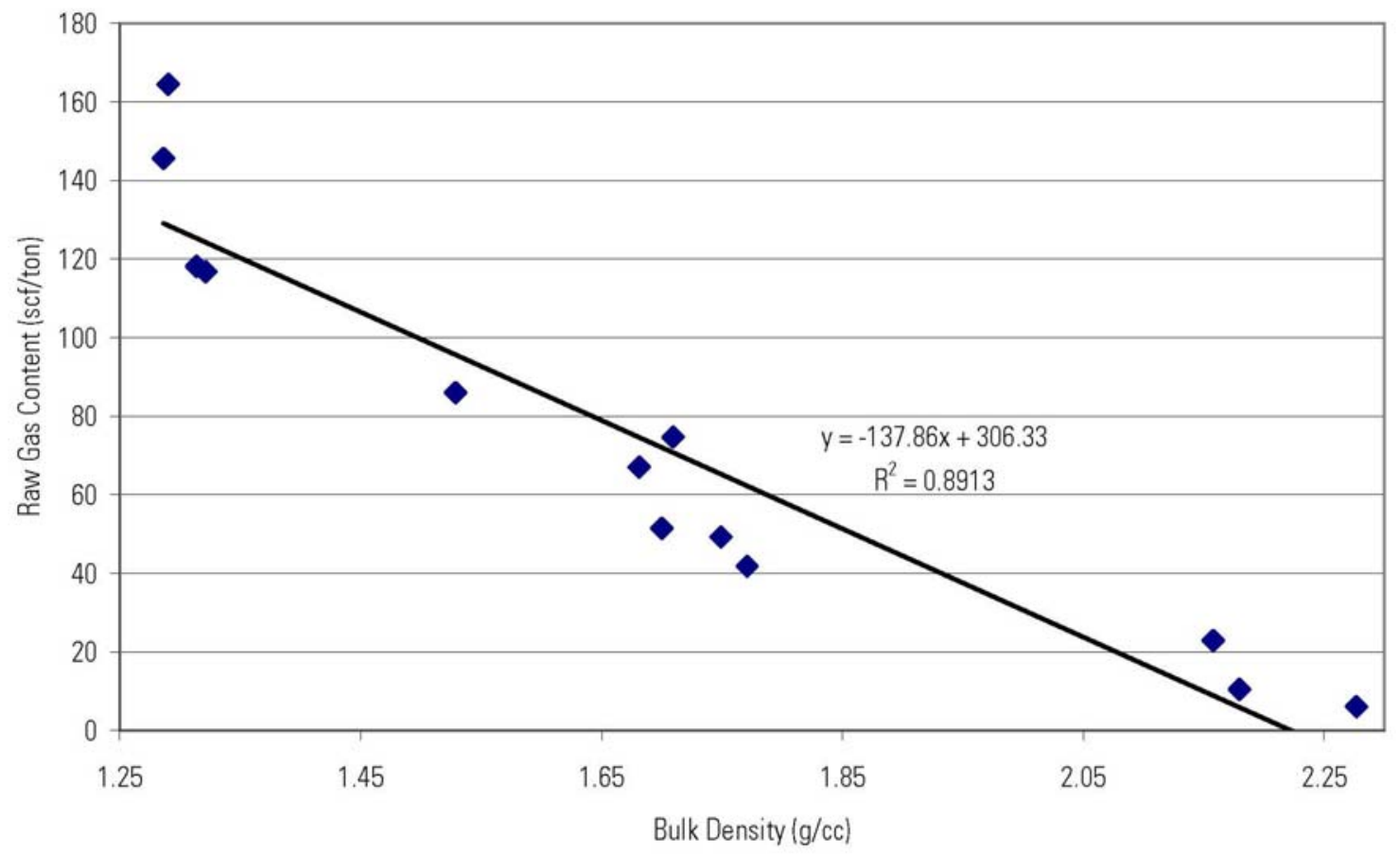

Figure 4. Raw gas content versus bulk density, CZ Fee A No. 114 CBM well samples. Abbreviations: $\mathrm{scf} / \mathrm{ton}=$ standard cubic feet per ton; $\mathrm{g} / \mathrm{cc}=$ grams per cubic centimeter . 

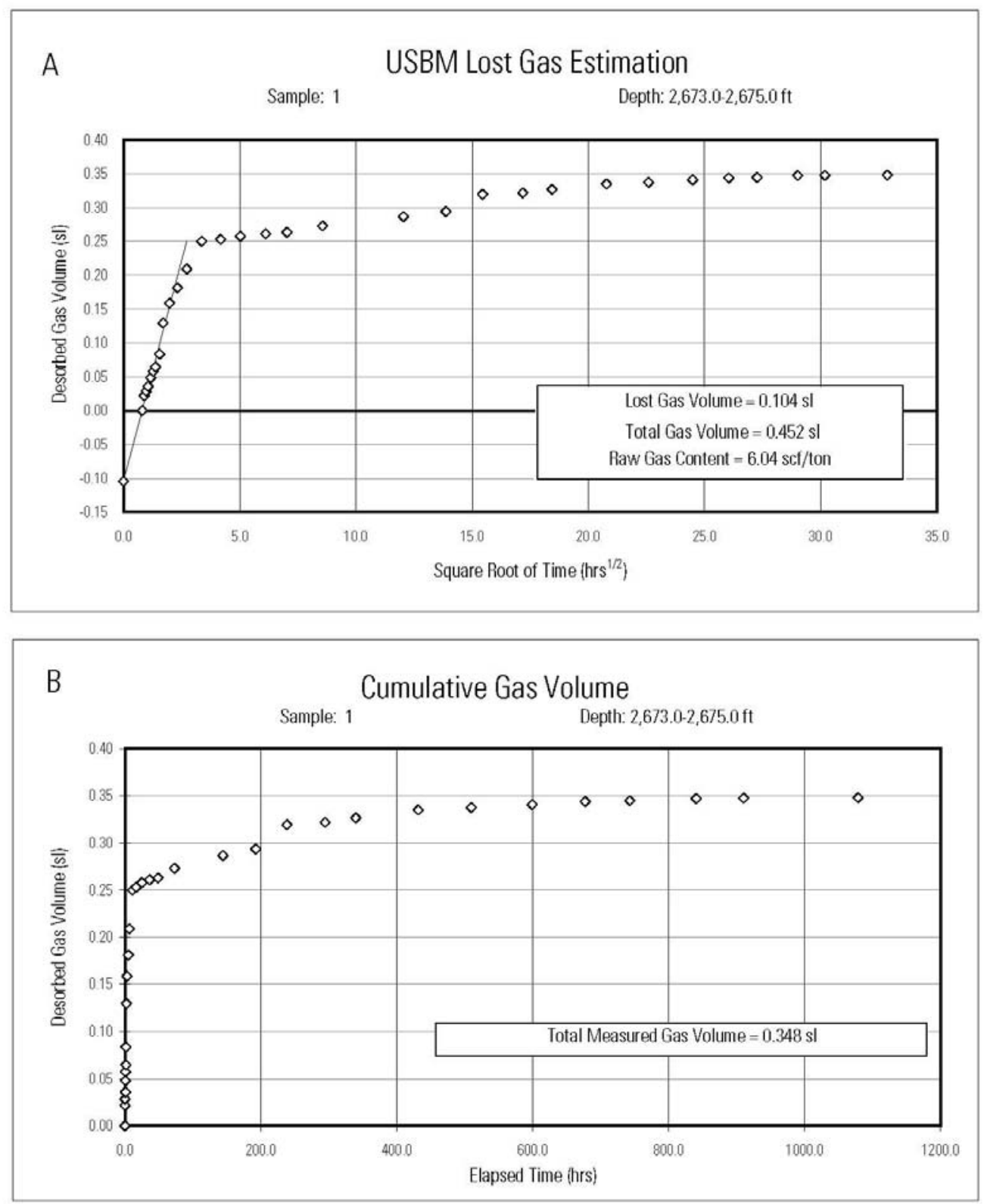

Figure 5. A. Lost gas estimation, and B. Cumulative gas volume for CZ Fee A No. 114 CBM well, sample 1. Abbreviations: $\mathrm{sl}=$ standard liter; scf/ton = standard cubic foot per ton; hrs = hours. Desorbed gas volume on abscissa ranges 0.00-0.40 sl. 

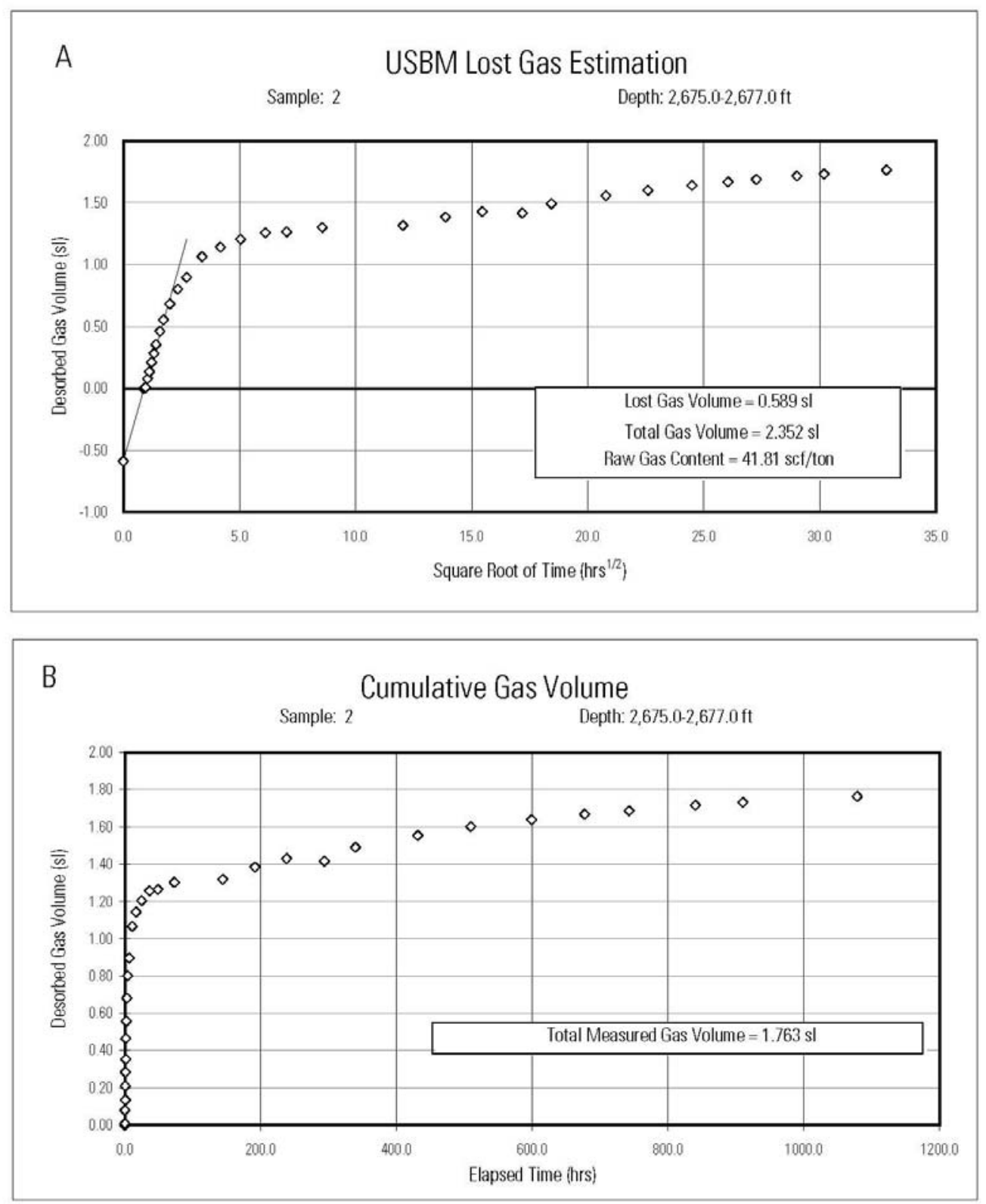

Figure 6. A. Lost gas estimation, and B. Cumulative gas volume for CZ Fee A No. 114 CBM well, sample 2. Abbreviations: $\mathrm{sl}=$ standard liter; scf/ton = standard cubic foot per ton; $\mathrm{hrs}=$ hours. Desorbed gas volume on abscissa ranges 0.00-2.00 sl. 

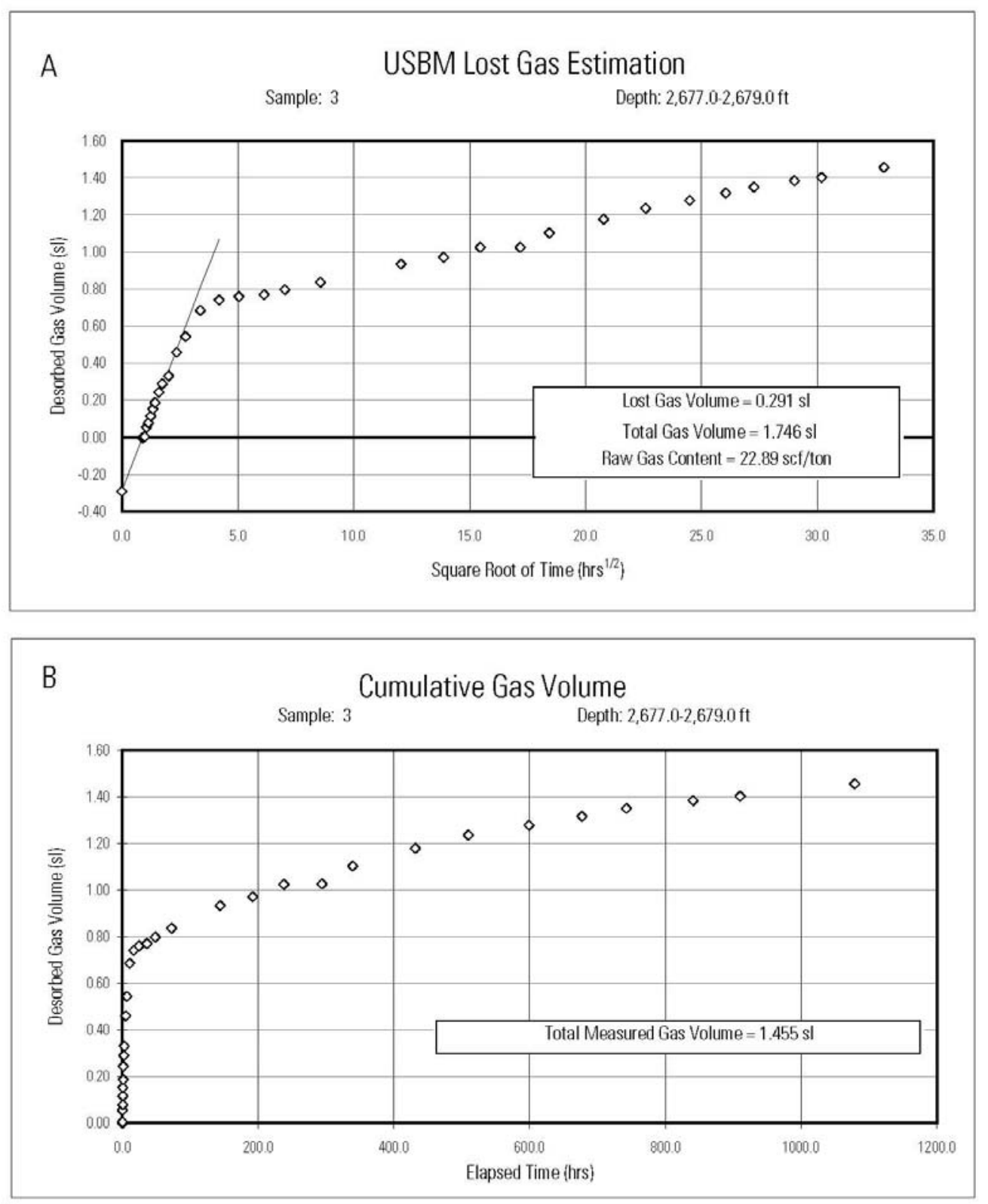

Figure 7. A. Lost gas estimation, and B. Cumulative gas volume for CZ Fee A No. 114 CBM well, sample 3. Abbreviations: $\mathrm{sl}=$ standard liter; scf/ton = standard cubic foot per ton; hrs = hours. Desorbed gas volume on abscissa ranges 0.00-1.60 sl. 

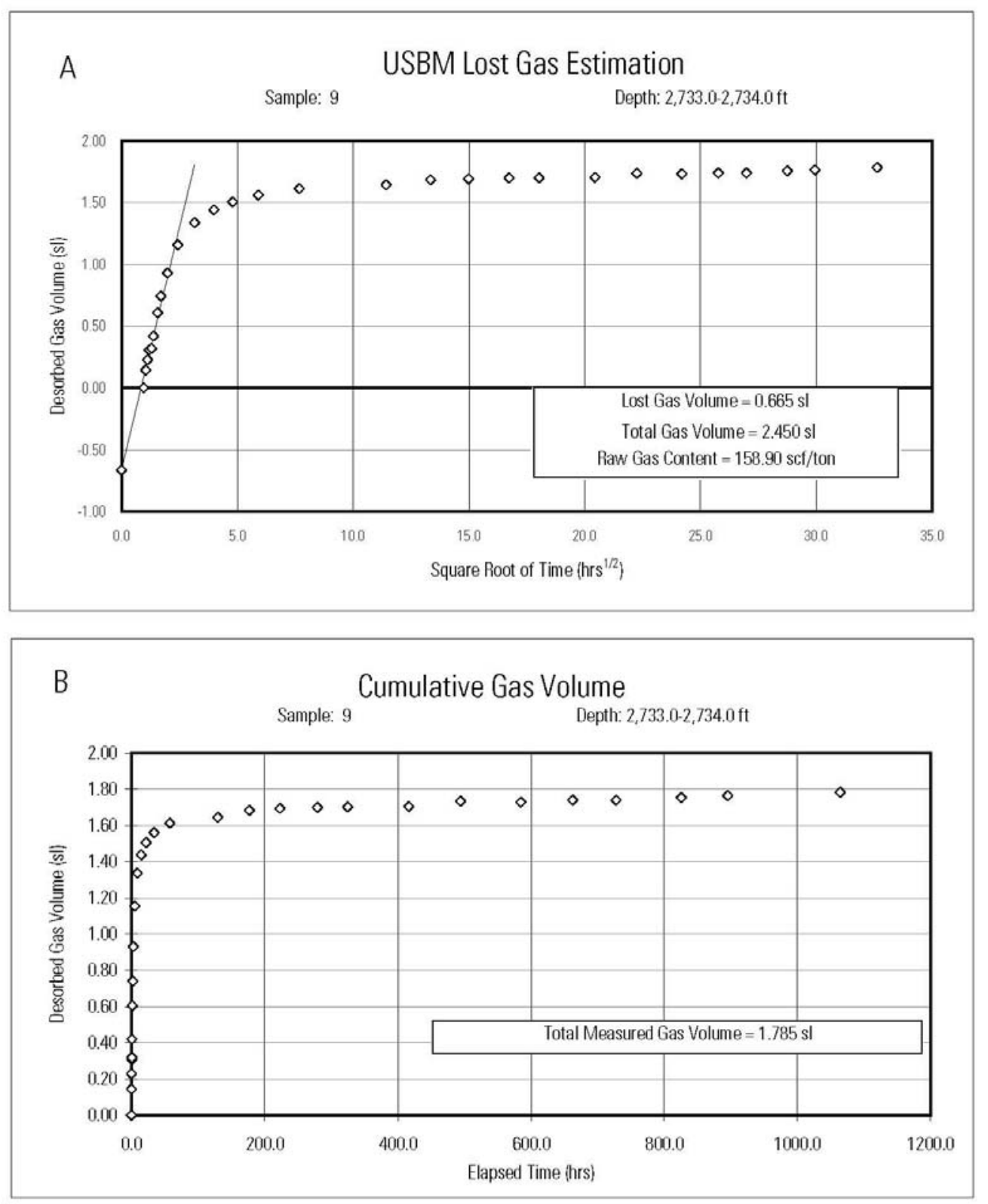

Figure 8. A. Lost gas estimation, and B. Cumulative gas volume for CZ Fee A No. 114 CBM well, sample 9. Abbreviations: $\mathrm{sl}=$ standard liter; scf/ton = standard cubic foot per ton; hrs = hours. Desorbed gas volume on abscissa ranges 0.00-2.00 sl. 

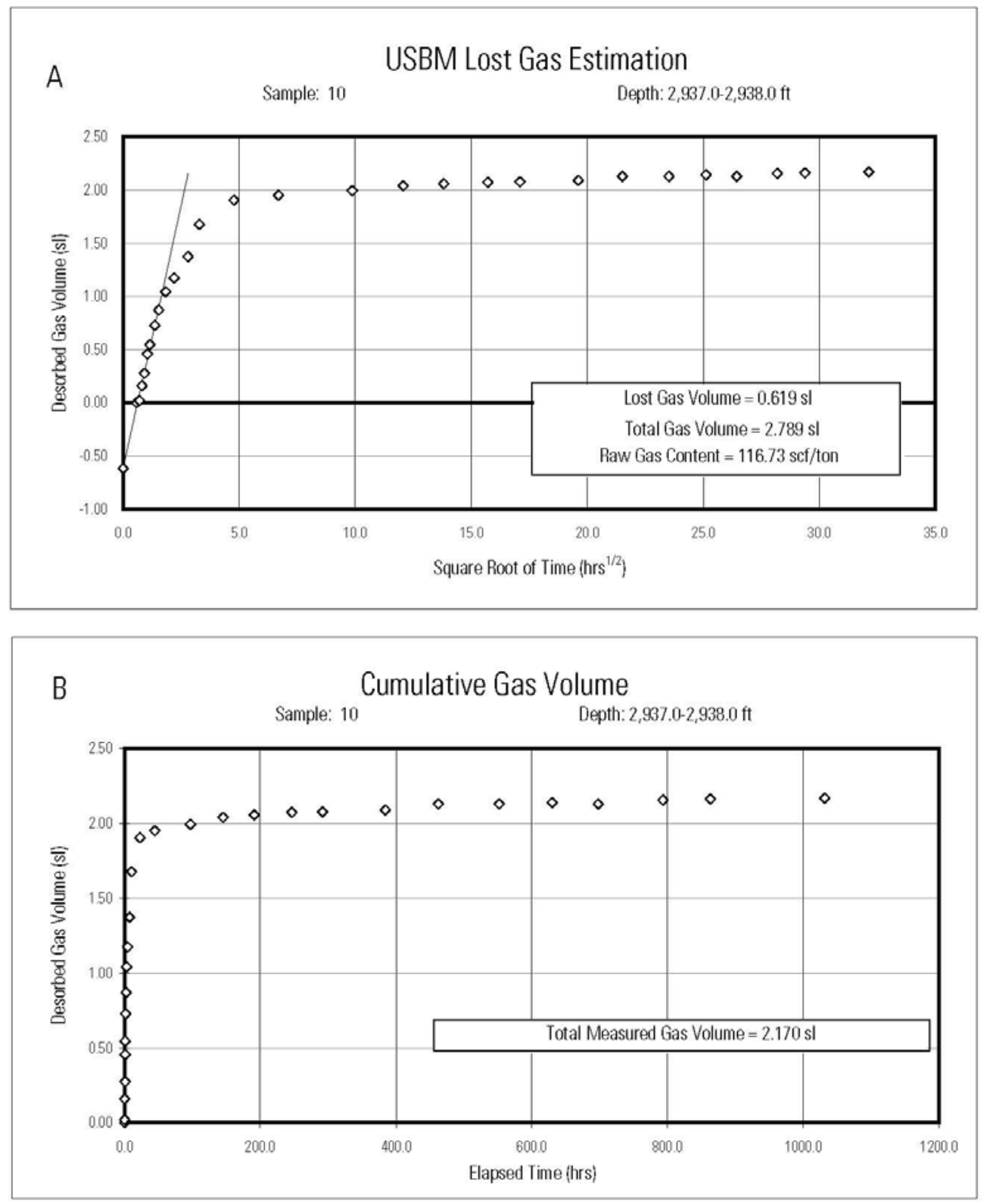

Figure 9. A. Lost gas estimation, and B. Cumulative gas volume for CZ Fee A No. 114 CBM well, sample 10. Abbreviations: $\mathrm{sl}=$ standard liter; scf/ton = standard cubic foot per ton; hrs = hours. Desorbed gas volume on abscissa ranges 0.00-2.50 sl. 

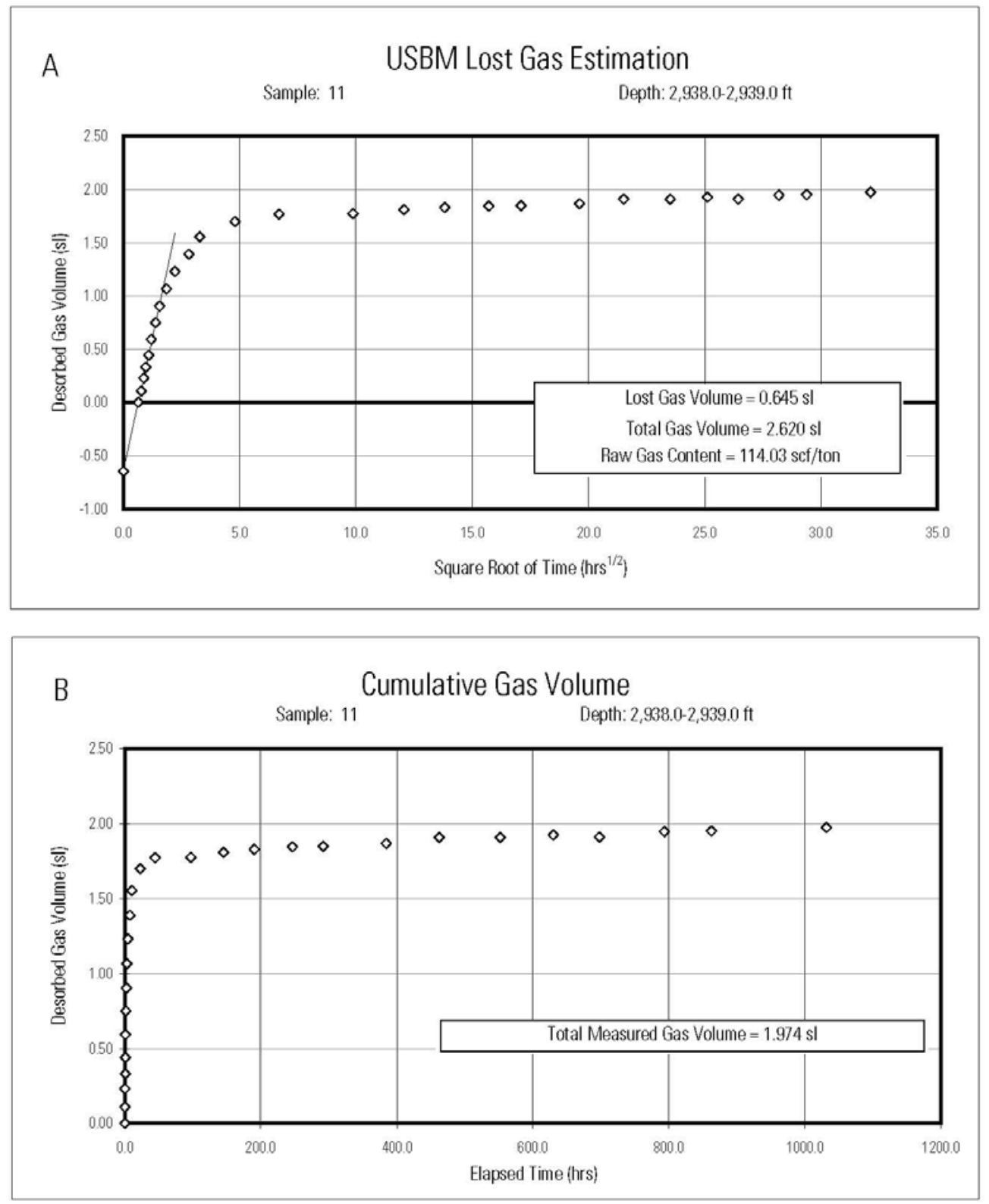

Figure 10. A. Lost gas estimation, and B. Cumulative gas volume for CZ Fee A No. 114 CBM well, sample 11. Abbreviations: $\mathrm{sl}=$ standard liter; scf/ton = standard cubic foot per ton; hrs = hours. Desorbed gas volume on abscissa ranges 0.00-2.50 sl. 

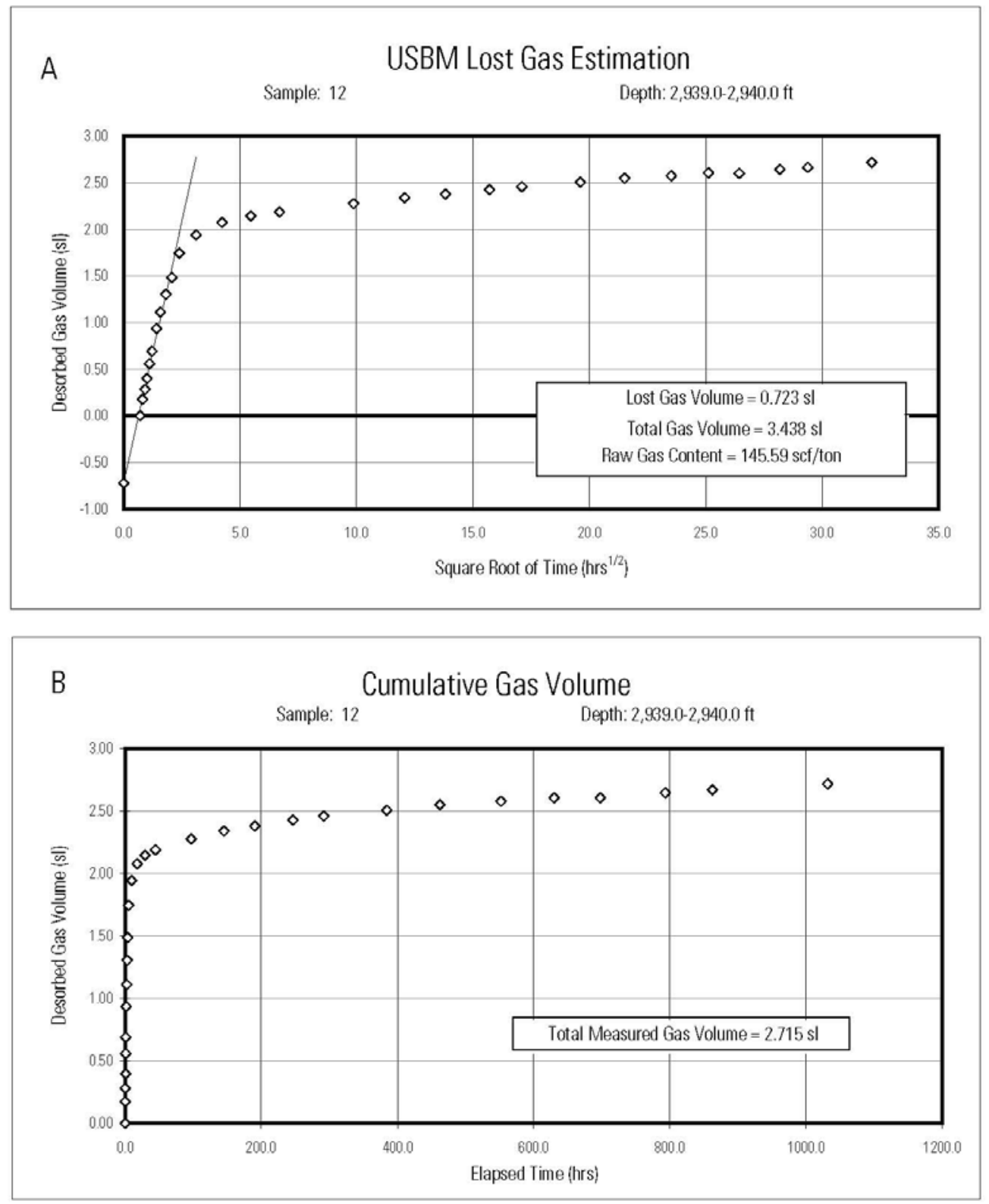

Figure 11. A. Lost gas estimation, and B. Cumulative gas volume for CZ Fee A No. 114 CBM well, sample 12. Abbreviations: $\mathrm{sl}=$ standard liter; scf/ton = standard cubic foot per ton; hrs = hours. Desorbed gas volume on abscissa ranges 0.00-3.00 sl. 

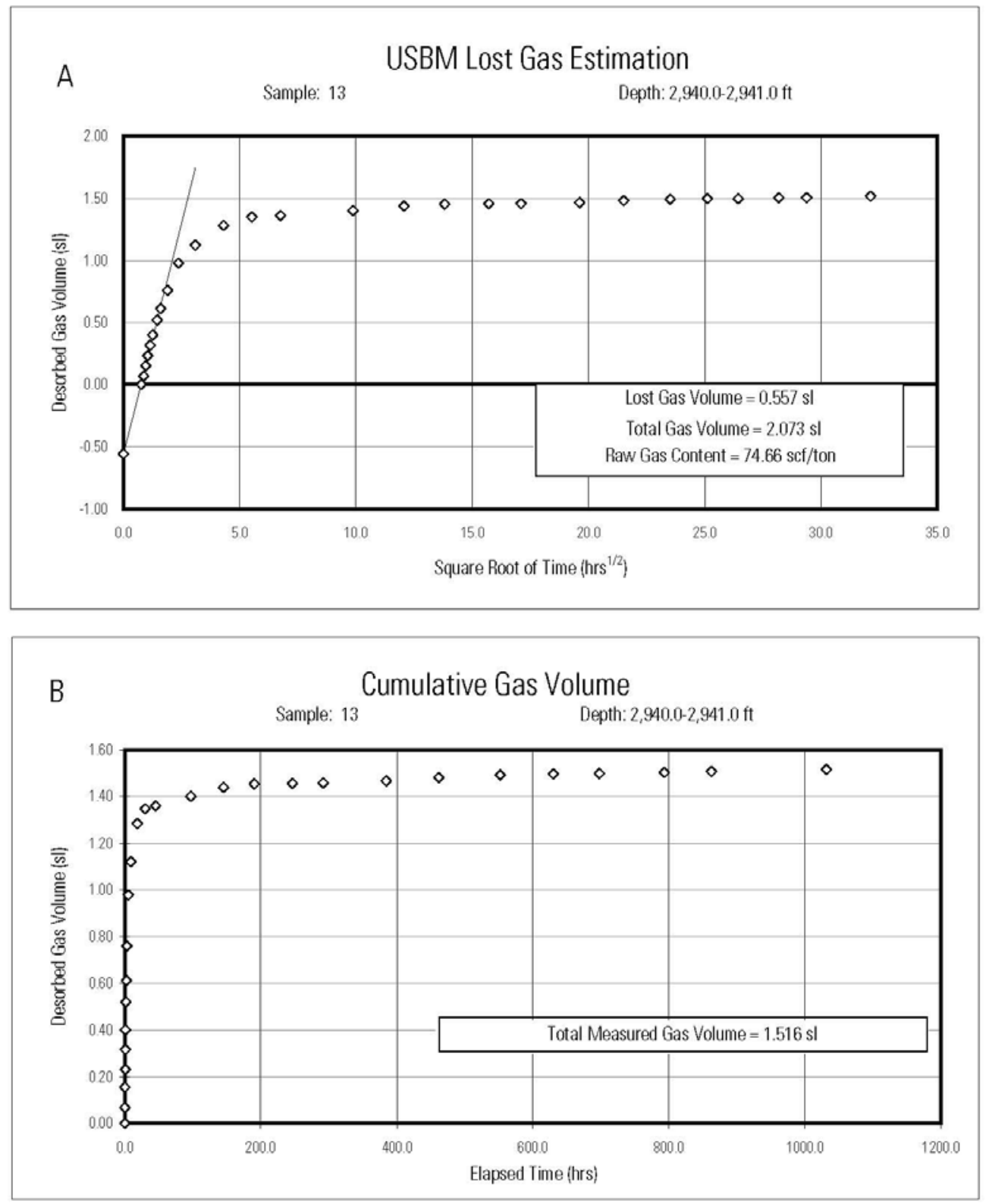

Figure 12. A. Lost gas estimation, and B. Cumulative gas volume for CZ Fee A No. 114 CBM well, sample 13. Abbreviations: $\mathrm{sl}=$ standard liter; scf/ton = standard cubic foot per ton; hrs = hours. Desorbed gas volume on abscissa ranges 0.00-1.60 sl. 


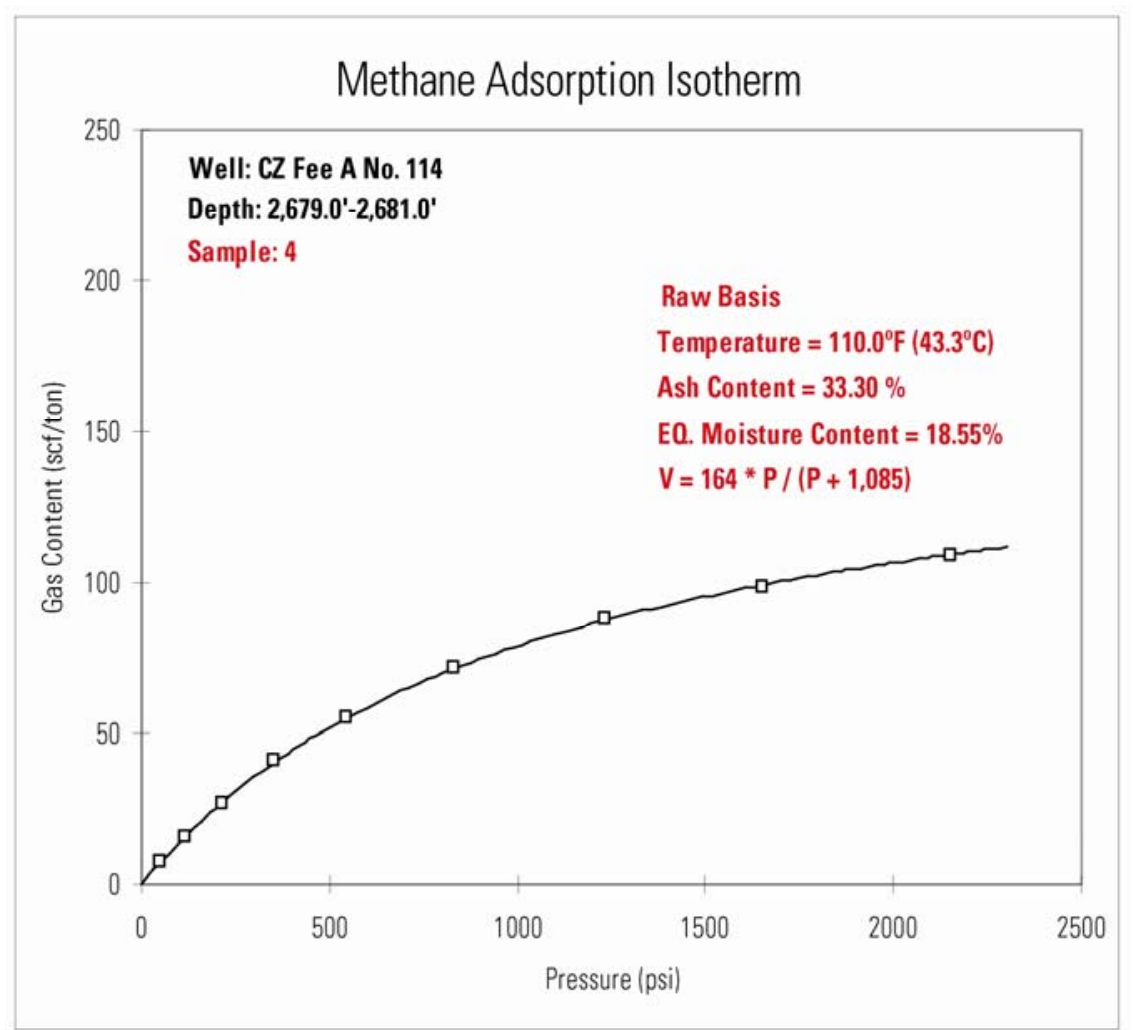

Figure 13. Methane adsorption isotherm, CZ Fee A No. 114 CBM well, sample 4, raw basis. Abbreviations: $E Q$. = equilibrium; $V=$ volume; $P=$ pressure; scf/ton = standard cubic feet per ton; $p s i=$ pounds per square inch.

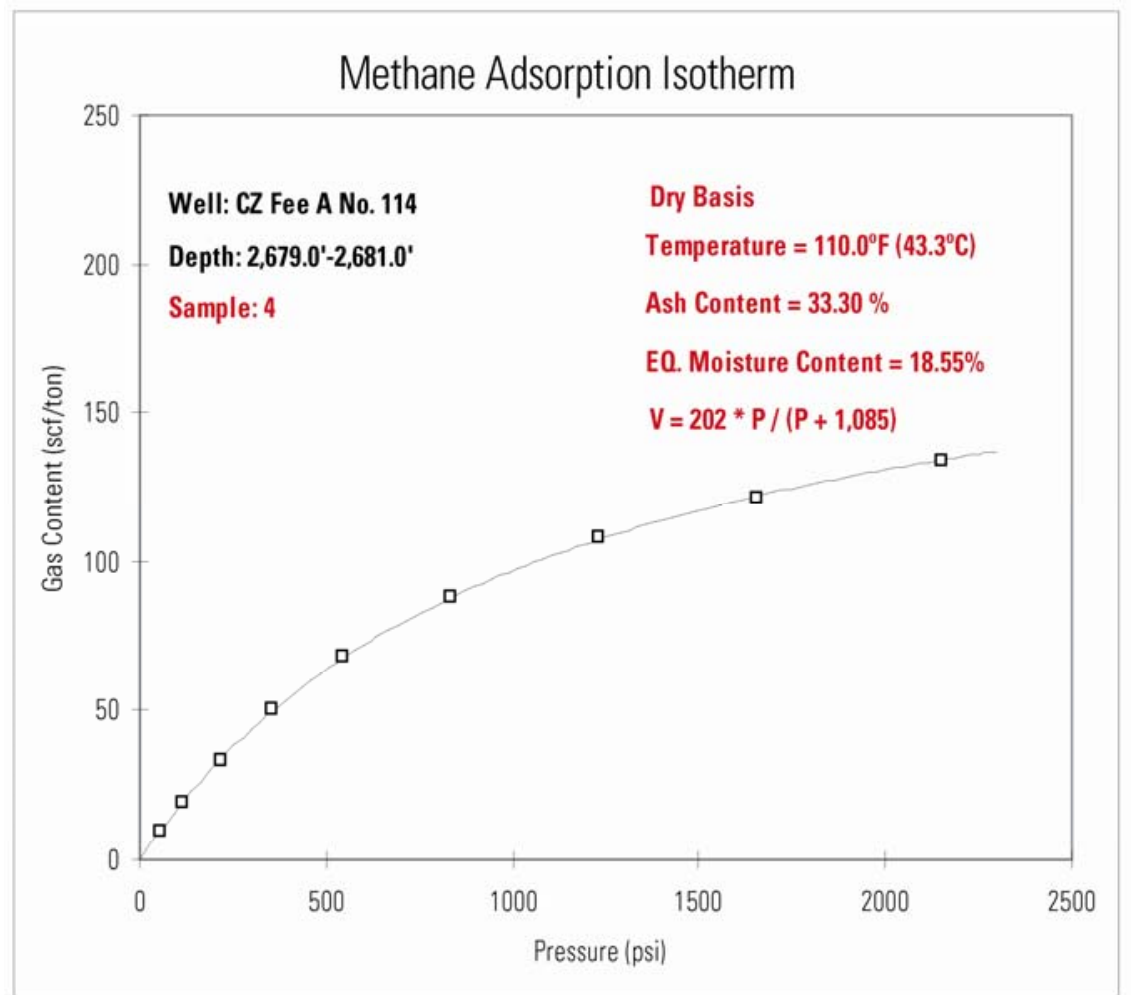

Figure 14. Methane adsorption isotherm, CZ Fee A No. 114 CBM well, sample 4, dry basis. Abbreviations: $\mathrm{EQ}$. = equilibrium; $\mathrm{V}=$ volume; $\mathrm{P}=$ pressure; $\mathrm{scf} / \mathrm{ton}=$ standard cubic feet per ton; $p s i=$ pounds per square inch. 


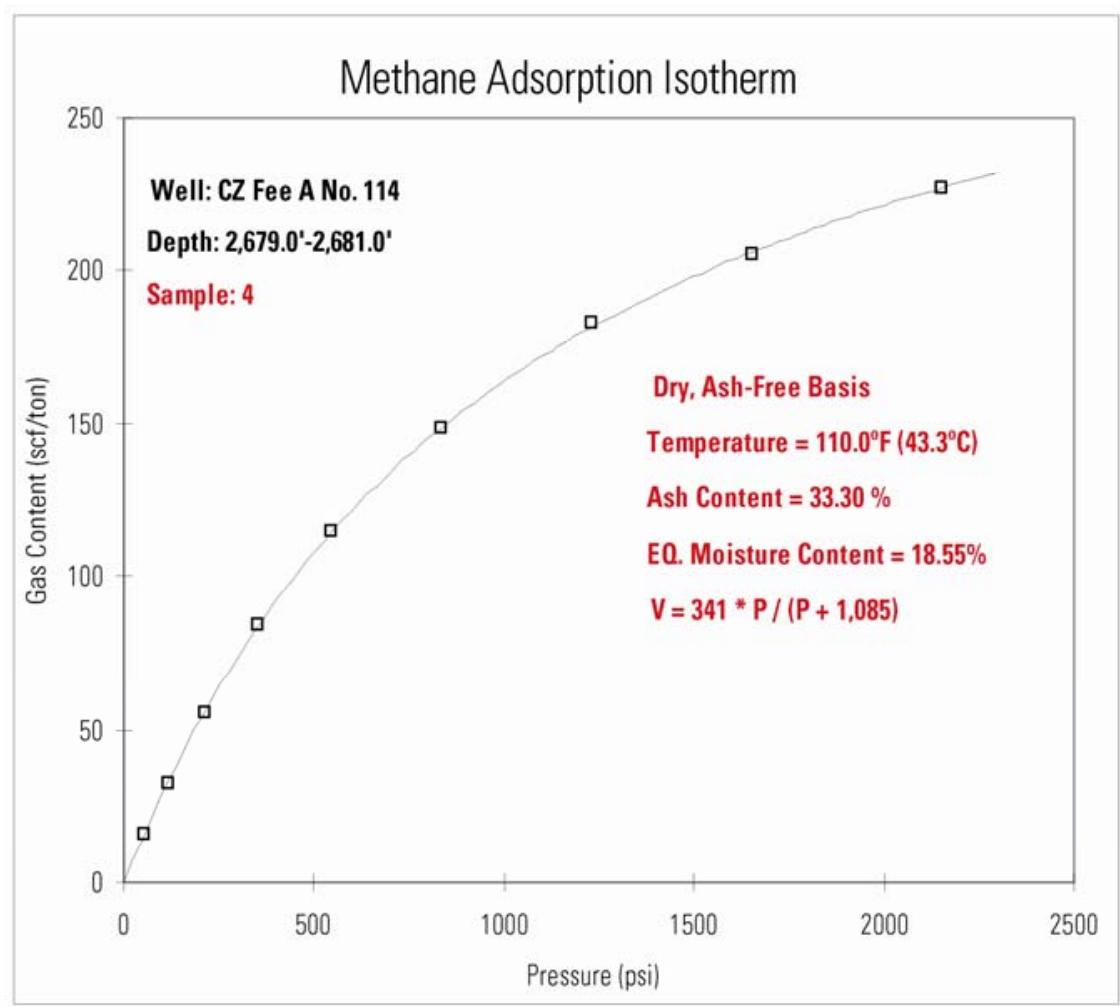

Figure 15. Methane adsorption isotherm, CZ Fee A No. 114 CBM well, sample 4, dry, ash-free basis. Abbreviations: $E Q$. = equilibrium; $V=$ volume; $P=$ pressure; scf $/$ ton = standard cubic feet per ton; $p s i=$ pounds per square inch.

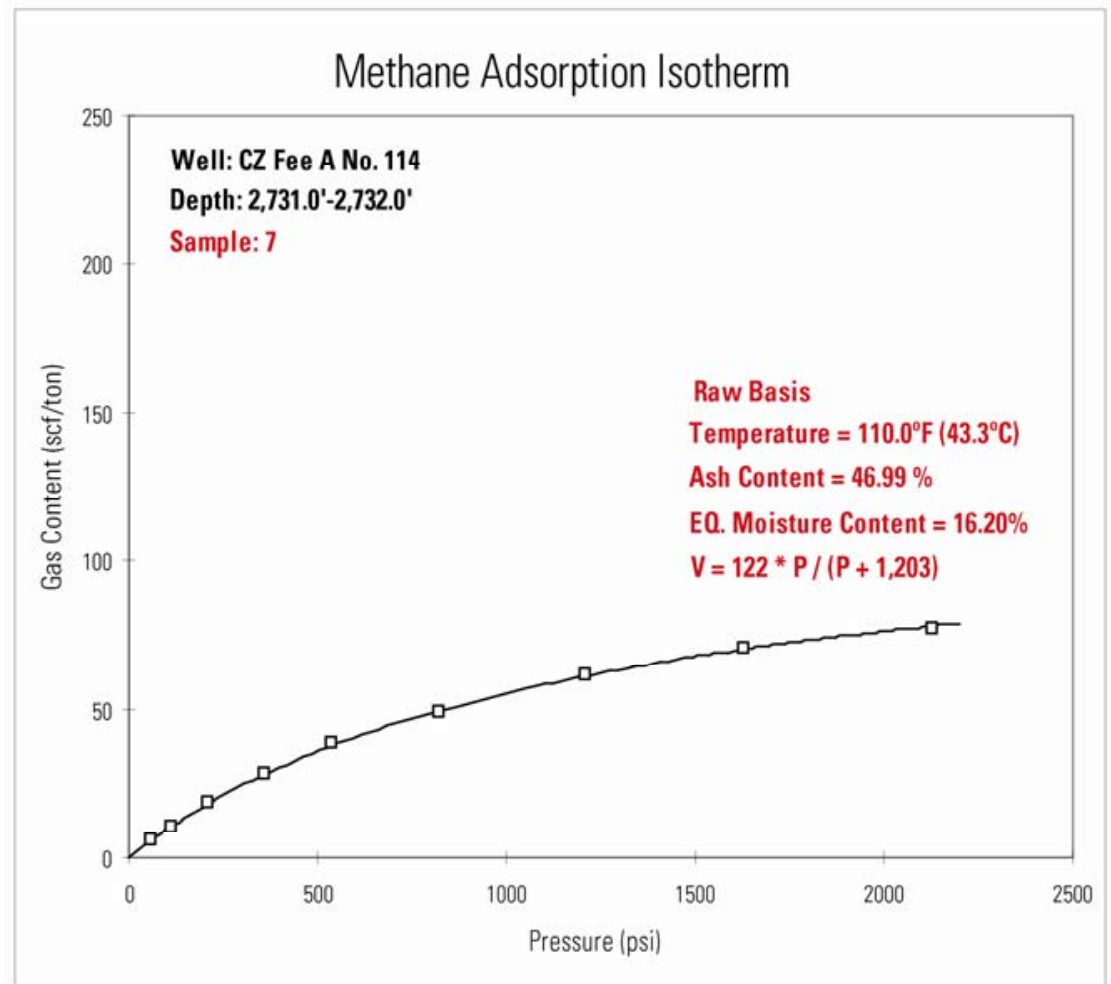

Figure 16. Methane adsorption isotherm, CZ Fee A No. 114 CBM well, sample 7, raw basis. Abbreviations: $E Q$. = equilibrium; $V=$ volume; $P=$ pressure; $s c f / t o n=$ standard cubic feet per ton; $p s i=$ pounds per square inch. 


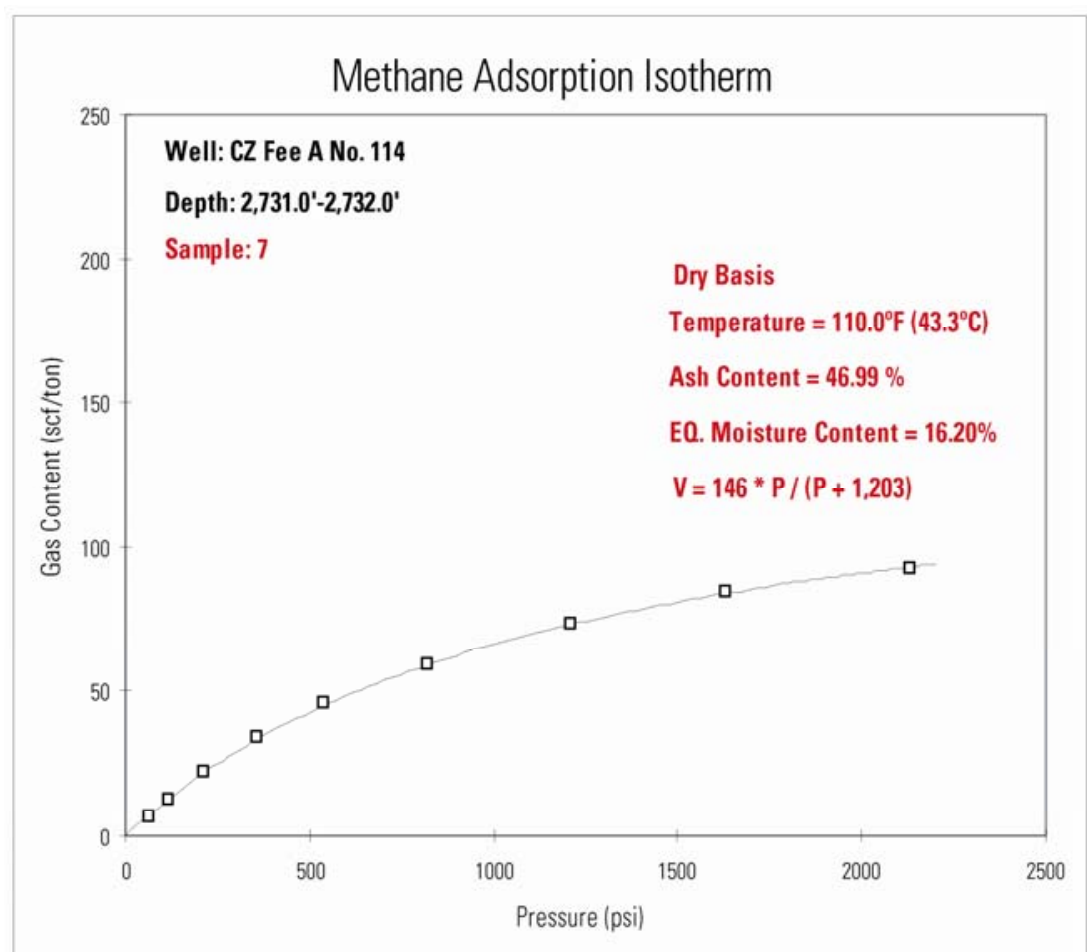

Figure 17. Methane adsorption isotherm, CZ Fee A No. 114 CBM well, sample 7, dry basis. Abbreviations: $E Q$. = equilibrium; $V$ = volume; $\mathrm{P}=$ pressure; scf/ton = standard cubic feet per ton; psi = pounds per square inch.

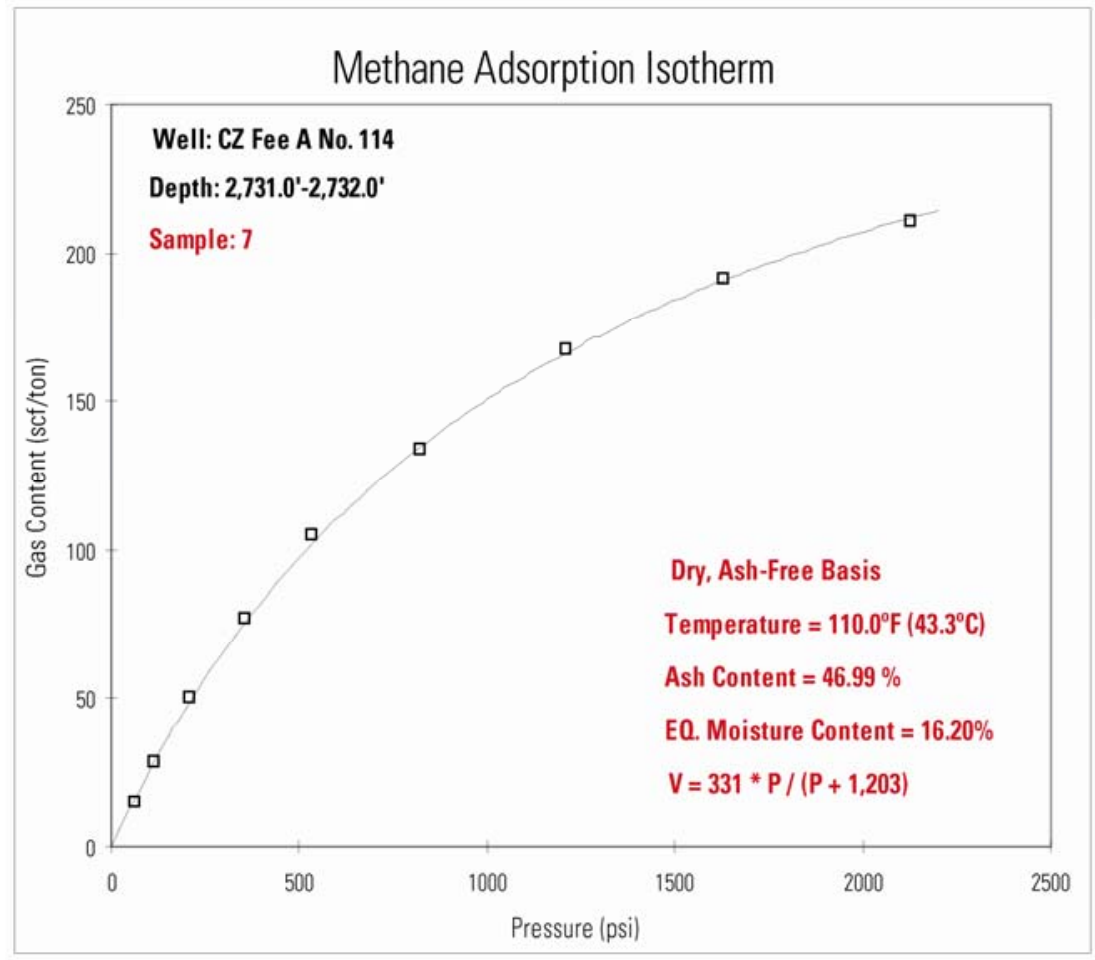

Figure 18. Methane adsorption isotherm, CZ Fee A No. 114 CBM well, sample 7, dry, ash-free basis. Abbreviations: $E Q$. = equilibrium; $V=$ volume; $P=$ pressure; scf/ton = standard cubic feet per ton; psi = pounds per square inch. 


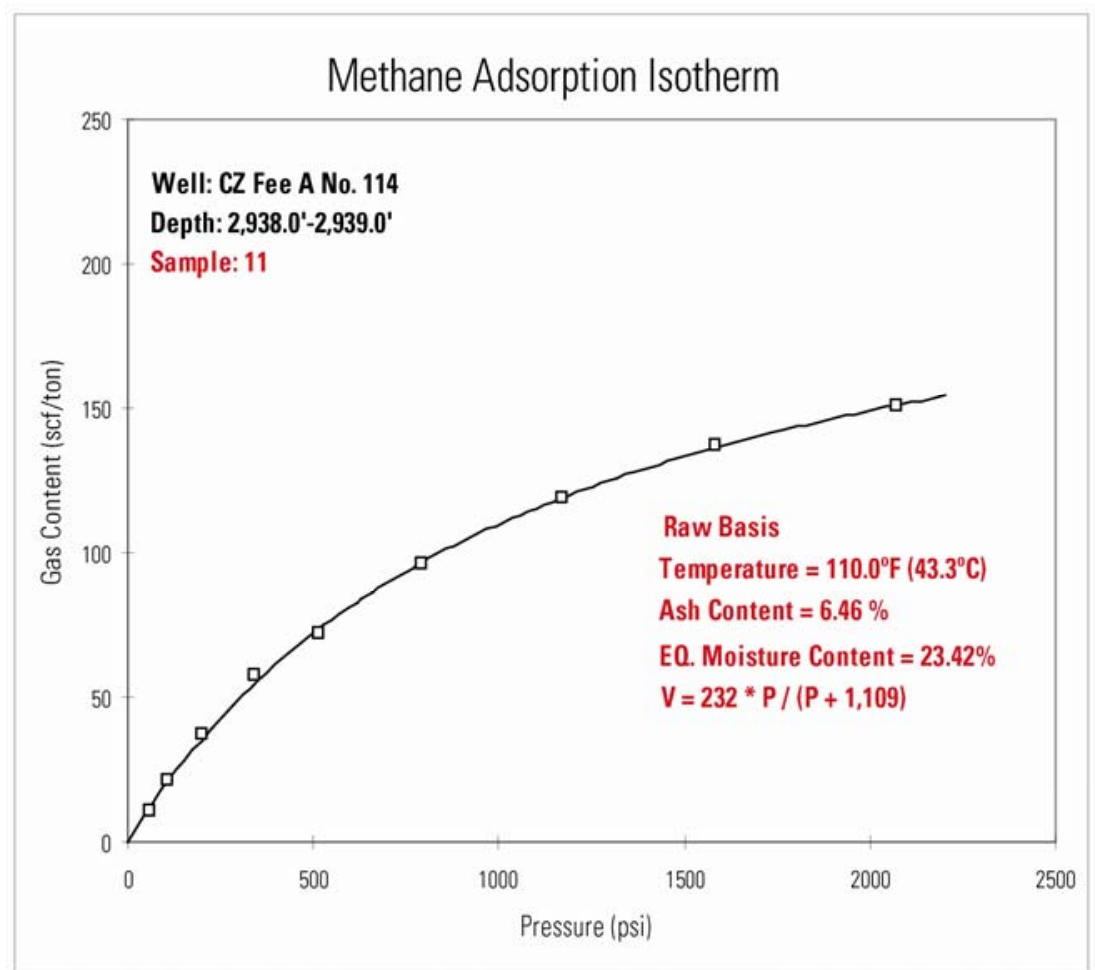

Figure 19. Methane adsorption isotherm, CZ Fee A No. 114 CBM well, sample 11, raw-basis. Abbreviations: $E Q$. = equilibrium; $V=$ volume; $P$ = pressure; scf/ton = standard cubic feet per ton; $p s i=$ pounds per square inch.

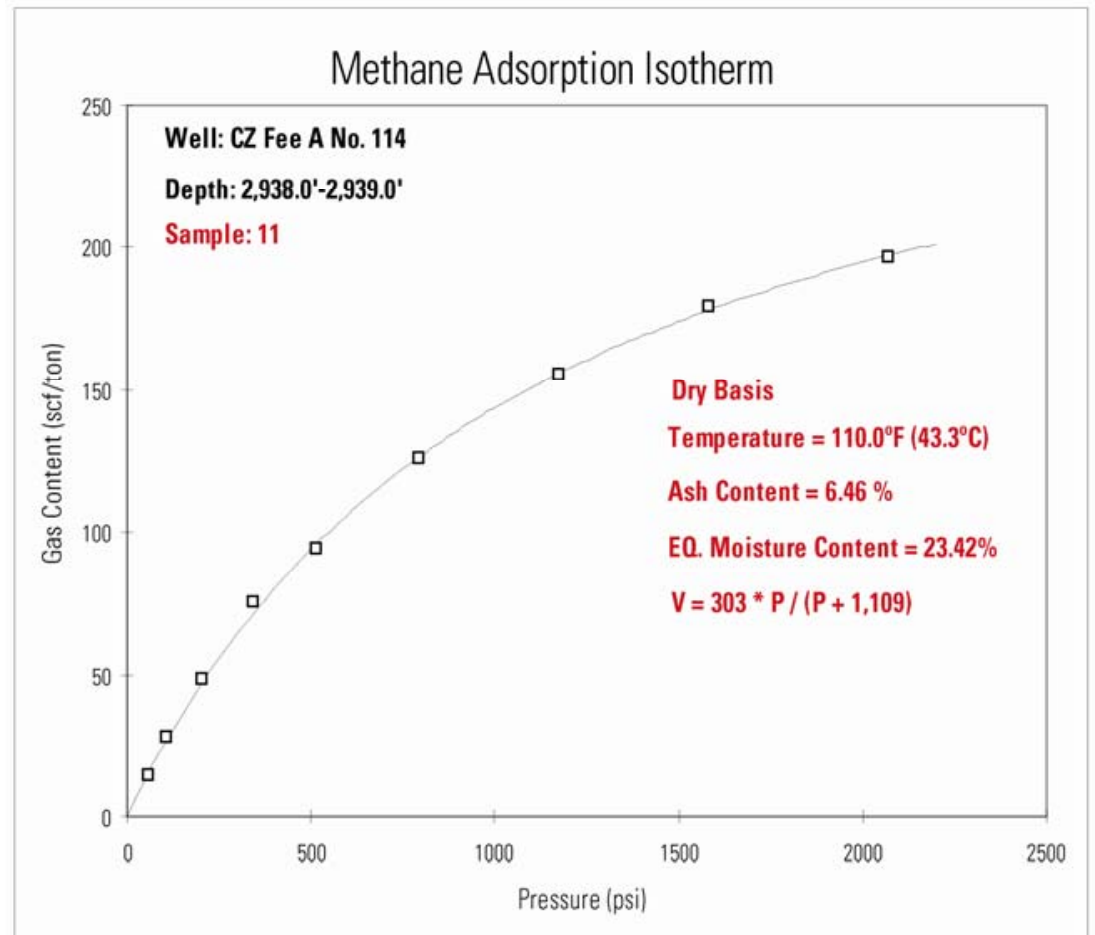

Figure 20. Methane adsorption isotherm, CZ Fee A No. 114 CBM well, sample 11, dry basis. Abbreviations: $\mathrm{EQ}$. = equilibrium; $\mathrm{V}=$ volume; $\mathrm{P}=$ pressure; scf/ton = standard cubic feet per ton; $p s i=$ pounds per square inch. 


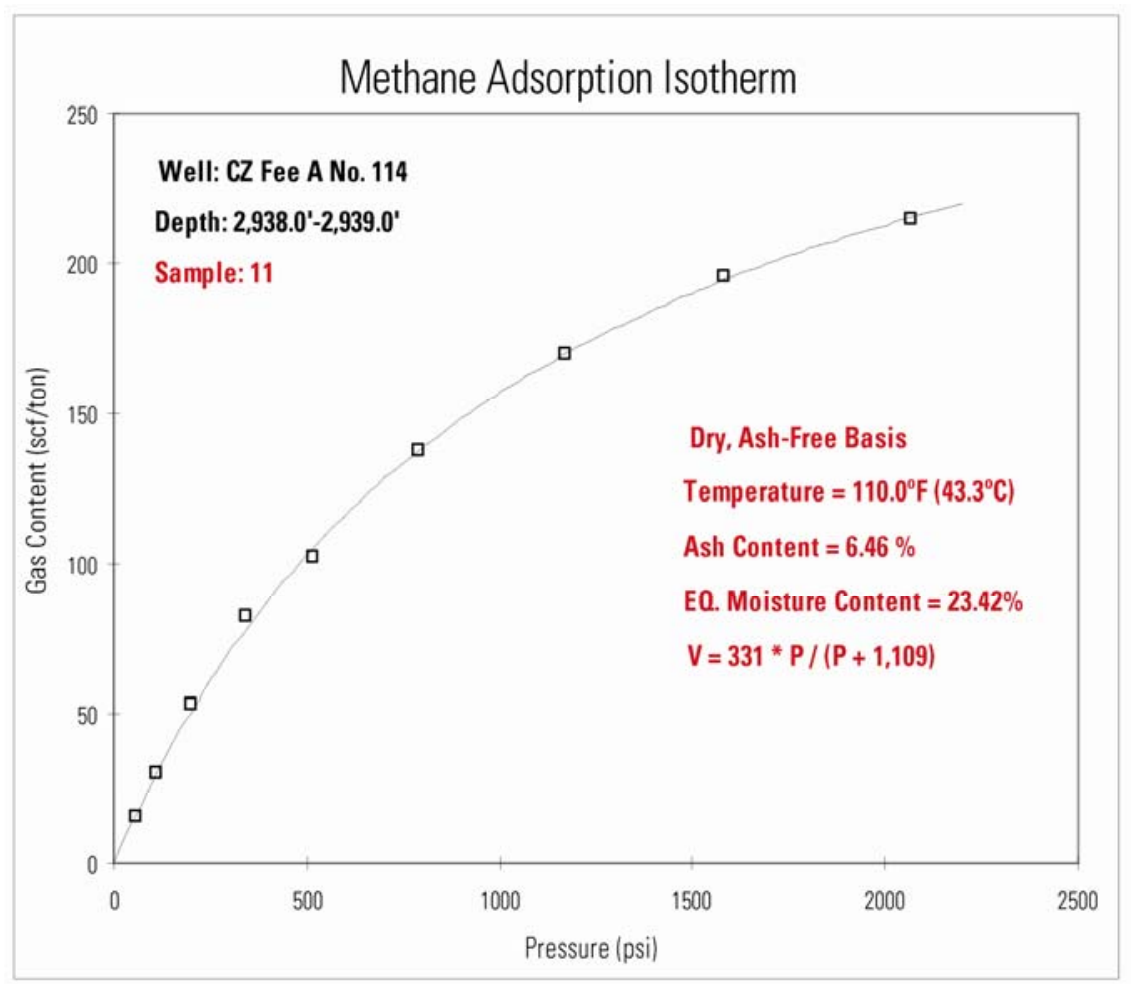

Figure 21. Methane adsorption isotherm, CZ Fee A No. 114 CBM well, sample 11, dry, ashfree basis. Abbreviations: $E Q$. = equilibrium; $V=$ volume; $P=$ pressure; scf $/$ ton = standard cubic feet per ton; psi = pounds per square inch. 


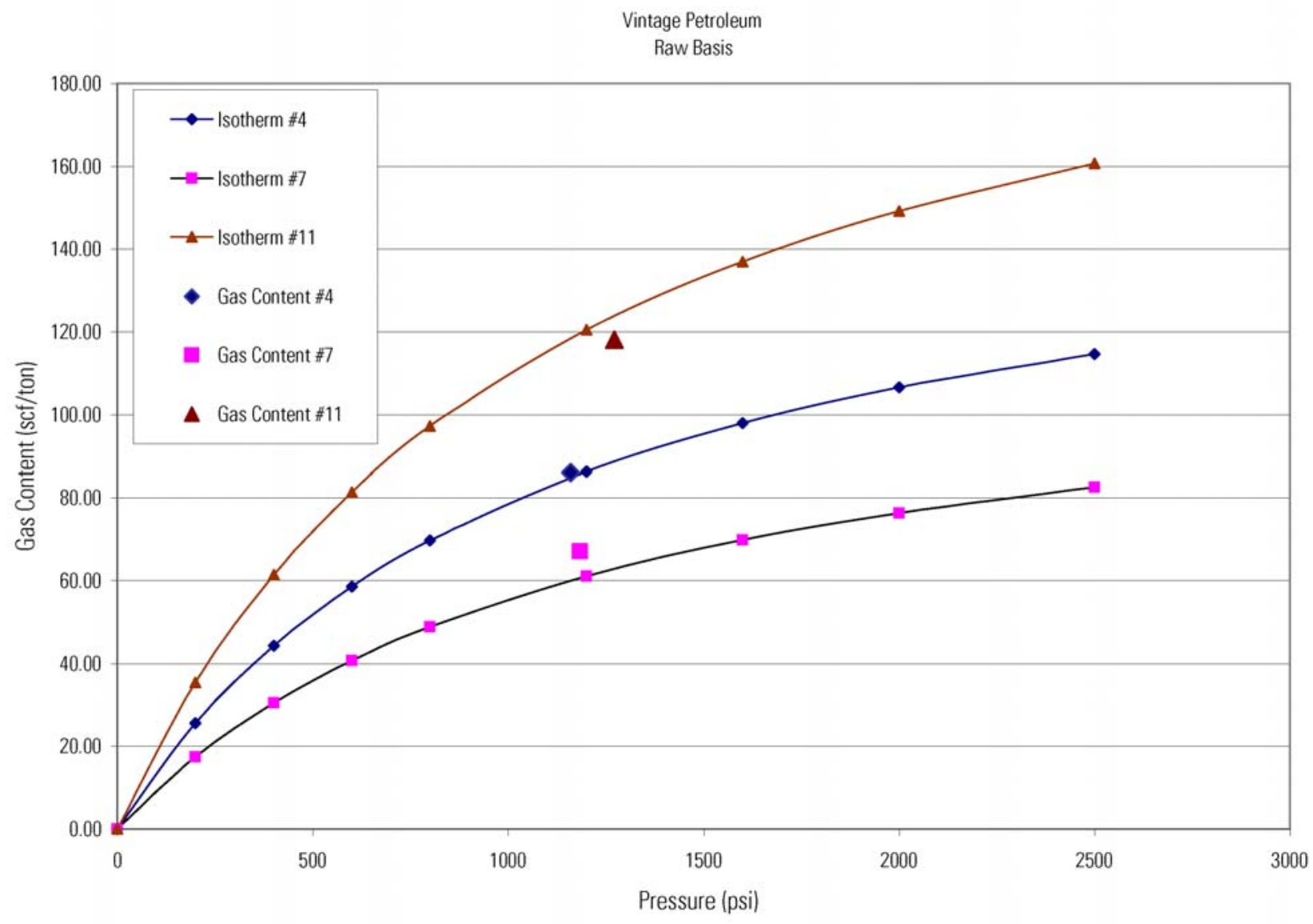

Figure 22. Methane adsorption isotherms and measured gas content, CZ Fee A No. 114 CBM well, as-analyzed basis. Abbreviations: scf/ton = standard cubic feet per ton; $p s i=$ pounds per square inch. 
Table 1. General information for CZ Fee A No. 114 CBM well, Winn Parish, LA.

Hole name: CZ Fee A No. 114

API number: 17127223830000

Louisiana serial number:

228951

Spud Date: $1 / 8 / 2004$

Completion Date: 4/12/2004

Operator: Vintage Petroleum,

Inc.

Parish: Winn

Latitude: $31^{\circ} 54^{\prime} 28^{\prime \prime}$

Longitude: $92^{\circ} 29^{\prime} 19^{\prime \prime}$

Township, Range, Section: 11N, 01W,

029

Perforation Interval: $2,730-2,734$

$\mathrm{ft}$

Total depth of hole:

$3,114 \mathrm{ft}$

Cored Intervals: 2,673-2,683 ft, 2,730-2,734 ft, 2,937-2,941 ft, all in Wilcox Group (PaleoceneEocene). 
Table 2. Gas desorption data for CZ Fee A No. 114

CBM well.

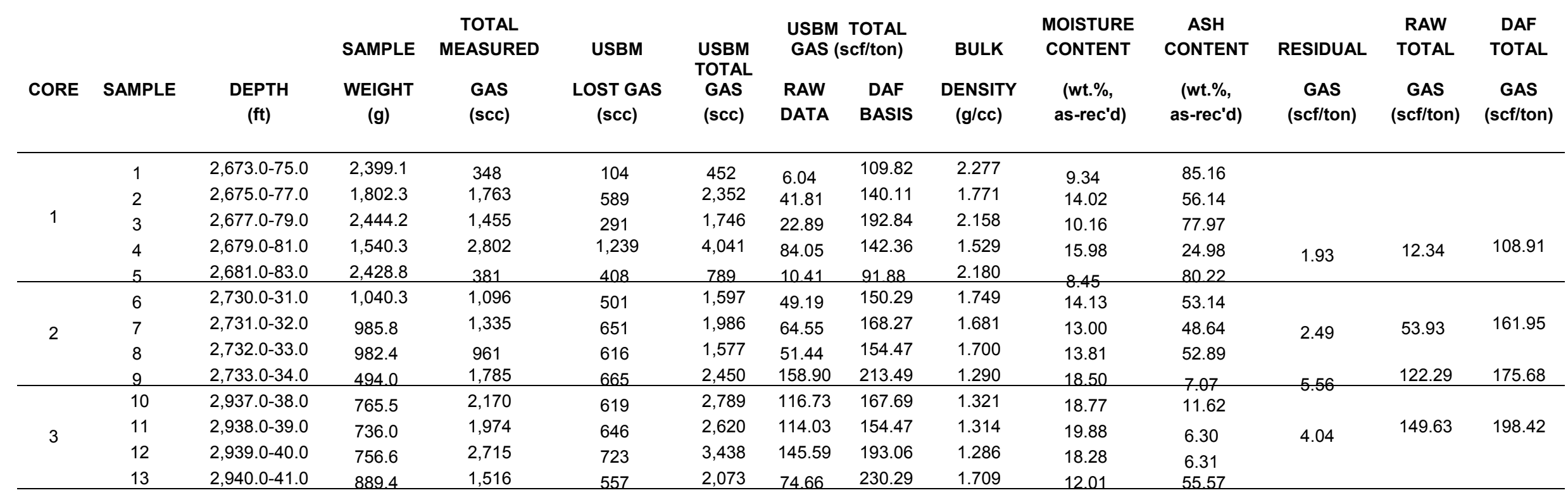

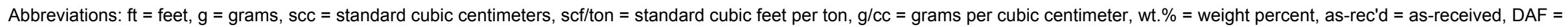
dry, ash-free, USBM = U.S. Bureau of Mines (Diamond and Levine, 1981). 
Table 3. Coal quality data for CZ Fee A No. 114 CBM well, sample 4 (2,679.0-2,681.0 ft).

ANALYSIS

\begin{tabular}{rrr} 
As- & Moisture- & Moisture \\
Received & Free & \& Ash-Free \\
$($ wt. \%) & $($ wt. \%) & $($ wt. \%) \\
\hline
\end{tabular}

PROXIMATE ANALYSIS

$\begin{array}{lrrr}\text { Moisture } & 13.88 & & \\ \text { Ash } & 35.25 & 40.93 & \\ \text { Volatile Matter } & 26.02 & 30.21 & 51.15 \\ \text { Fixed Carbon } & 24.85 & 28.86 & 48.85 \\ \text { Total } & 100.00 & 100.00 & 100.00 \\ \text { HEATING VALUE (Btu/lb) } & & & \\ \text { SULFUR } & 6,624 & 7,692 & 13,021 \\ & & & \\ & 0.99 & 1.15 & 1.95\end{array}$

COAL RANKING DATA -- BASED ON PARR FORMULAS (ASTM D 388)

Dry, Mm-free FC

Dry, Mm-free VM

Moist, Mm-free Btu

Agglomerating Characteristics

Coal Rank
52.00

48.00

10,710

Not Determined

Sub Bituminous

Abbreviations: $\mathrm{ft}=$ feet, $\mathrm{wt} . \%=$ weight percent, $\mathrm{Btu} / \mathrm{lb}=$ British thermal units per pound, $\mathrm{FC}=$ fixed carbon, $\mathrm{VM}=$ volatile matter, $\mathrm{Mm}=$ mineral matter, $\mathrm{ASTM}=$ American Society for Testing and Materials (ASTM, 2005). 
Table 4. Coal quality data for CZ Fee A No. 114 CBM well, sample 7 (2,731.0-2,732.0 ft).

ANALYSIS

As-

Received

Moisture-

Free

Moisture

(wt. \%)

(wt. \%)

\& Ash-Free

(wt. \%)

PROXIMATE ANALYSIS

Moisture

Ash

12.13

47.41

22.99

Volatile Matter

17.47

Fixed Carbon

100.00

53.95

26.16

19.88

56.82

Total

100.00

43.18

100.00

HEATING VALUE (Btu/lb)

4,899

5,575

12,108

SULFUR

0.56

0.64

1.38

COAL RANKING DATA -- BASED ON PARR FORMULAS (ASTM D 388)

Dry, Mm-free FC

Dry, Mm-free VM

Moist, Mm-free Btu

Agglomerating Characteristics

Coal Rank
47.82

52.18

10,046

Not Determined

Sub Bituminous

Abbreviations: $\mathrm{ft}=$ feet, $\mathrm{wt} . \%=$ weight percent, $\mathrm{Btu} / \mathrm{lb}=$ British thermal units per pound, $\mathrm{FC}=$ fixed carbon, $\mathrm{VM}=$ volatile matter, $\mathrm{Mm}=$ mineral matter, $\mathrm{ASTM}=$ American Society for Testing and Materials $($ ASTM, 2005). 
Table 5. Coal quality data for CZ Fee A No. 114 CBM well, sample 11 (2,938.0-2,939.0 $\mathrm{ft})$.

ANALYSIS

\begin{tabular}{rrr} 
As- & Moisture- & Moisture \\
Received & Free & \& Ass-Free \\
(wt. \%) & (wt. \%) & (wt. \%) \\
\hline
\end{tabular}

PROXIMATE ANALYSIS

Moisture

Ash

Volatile Matter

Fixed Carbon

Total

HEATING VALUE (Btu/lb)

SULFUR
18.65

6.29

33.21

41.85

100.00

10,151

1.26

\subsection{3}

40.82

51.44

100.00

12,478

1.55
44.24

55.76

100.00

13,524

1.68

COAL RANKING DATA -- BASED ON PARR FORMULAS (ASTM D 388)

$\begin{array}{lr}\text { Dry, Mm-free FC (wt.\%) } & 56.40 \\ \text { Dry, Mm-free VM (wt.\%) } & 43.60 \\ \text { Moist, Mm-free Btu/lb } & 10,904 \\ \text { Agglomerating Characteristics } & \text { Not Determined } \\ \text { Coal Rank } & \text { Sub Bituminous } \\ & \text { A }\end{array}$

Abbreviations: $\mathrm{ft}=$ feet, $\mathrm{wt} . \%=$ weight percent, $\mathrm{Btu} / \mathrm{lb}=$ British thermal units per pound, $\mathrm{FC}=$ fixed carbon, $\mathrm{VM}=$ volatile matter, $\mathrm{Mm}=$ mineral matter, $\mathrm{ASTM}=$ American Society for Testing and Materials (ASTM, 2005). 
Table 6. Petrographic data for CZ Fee A No. 114 CBM well, sample 4 (2,679.0-2,681.0 ft).

Maceral Type
Detrovitrinite
Telovitrinite
Total Vitrinite
Sporinite
Cutinite
Resinite
Exsudanite
(bitumen)
Total Liptinite
Detroinertinite
Fusinite
Semifusinite
Macrinite
Funginite
Total Inertinite
clay/other
quartz
carbonate
pyrite
Total Minerals
Total Sum

$\begin{array}{ccc}\text { Counts } & \begin{array}{c}\text { Maceral \% with } \\ \text { Mineral Matter }\end{array} & \begin{array}{c}\text { Maceral \% without } \\ \text { Mineral Matter }\end{array} \\ 344 & 34.57 \% & 69.49 \% \\ 78 & 7.84 \% & 15.76 \% \\ & \mathbf{4 2 . 4 1 \%} & \mathbf{8 5 . 2 5 \%} \\ 11 & 1.11 \% & 2.22 \% \\ 7 & 0.70 \% & 1.41 \% \\ 11 & 1.11 \% & 2.22 \% \\ & & \\ & 0.00 \% & 0.00 \% \\ 25 & \mathbf{2 . 9 1 \%} & \mathbf{5 . 8 6 \%} \\ 5 & 2.51 \% & 5.05 \% \\ 7 & 0.50 \% & 1.01 \% \\ 6 & 0.70 \% & 1.41 \% \\ 1 & 0.60 \% & 1.21 \% \\ & 0.10 \% & 0.20 \% \\ 500 & \mathbf{4 . 4 2 \%} & \mathbf{8 . 8 9 \%} \\ & 50.25 \% & \\ & 0.00 \% & \\ & 0.00 \% & \\ 995 & 0.00 \% & \\ & \mathbf{5 0 . 2 5 \%} & \end{array}$


Table 7. Petrographic data for CZ Fee A No. 114 CBM well, sample 7 (2,731.0-2,732.0 ft).

$\begin{array}{lccc}\text { Maceral Type } & \text { Counts } & \begin{array}{c}\text { Maceral \% with } \\ \text { Mineral Matter }\end{array} & \begin{array}{c}\text { Maceral \% without } \\ \text { Mineral Matter }\end{array} \\ \text { Detrovitrinite } & 330 & 33.92 \% & 69.77 \% \\ \text { Telovitrinite } & 75 & 7.71 \% & 15.86 \% \\ \text { Total Vitrinite } & & \mathbf{4 1 . 6 2 \%} & \mathbf{8 5 . 6 2 \%} \\ \text { Sporinite } & 8 & 0.82 \% & 1.69 \% \\ \text { Cutinite } & 1 & 0.10 \% & 0.21 \% \\ \text { Resinite } & 42 & 4.32 \% & 8.88 \% \\ \text { Exsudanite } & & & \\ \text { bitumen) } & 0 & 0.00 \% & 0.00 \% \\ \text { Total Liptinite } & & \mathbf{5 . 2 4 \%} & \mathbf{1 0 . 7 8 \%} \\ \text { Detroinertinite } & 11 & 1.13 \% & 2.33 \% \\ \text { Fusinite } & 1 & 0.10 \% & 0.21 \% \\ \text { Semifusinite } & 1 & 0.10 \% & 0.21 \% \\ \text { Macrinite } & 4 & 0.41 \% & 0.85 \% \\ \text { Funginite } & 0 & 0.00 \% & 0.00 \% \\ \text { Total Inertinite } & & \mathbf{1 . 7 5 \%} & \mathbf{3 . 5 9 \%} \\ \text { clay/other } & 500 & 51.39 \% & \\ \text { quartz } & 0 & 0.00 \% & \\ \text { carbonate } & 0 & 0.00 \% & \mathbf{1 0 0 . 0 0 \%} \\ \text { pyrite } & 0 & 0.00 \% & \\ \text { Total Minerals } & & \mathbf{5 1 . 3 9 \%} & \\ \text { Total Sum } & 973 & \mathbf{1 0 0 . 0 0 \%} & \end{array}$


Table 8. Petrographic data for CZ Fee A No. 114 CBM well, sample 11 (2,938.0-2,939.0 ft).

$\begin{array}{lccc}\text { Maceral Type } & \text { Counts } & \begin{array}{c}\text { Maceral \% with } \\ \text { Mineral Matter }\end{array} & \begin{array}{c}\text { Maceral \% without } \\ \text { Mineral Matter }\end{array} \\ \text { Detrovitrinite } & 251 & 46.14 \% & 47.81 \% \\ \text { Telovitrinite } & 78 & 14.34 \% & 14.86 \% \\ \text { Total Vitrinite } & & \mathbf{6 0 . 4 8 \%} & \mathbf{6 2 . 6 7 \%} \\ \text { Sporinite } & 15 & 2.76 \% & 2.86 \% \\ \text { Cutinite } & 2 & 0.37 \% & 0.38 \% \\ \text { Resinite } & 4 & 0.74 \% & 0.76 \% \\ \text { Exsudanite } & & & \\ \text { (bitumen) } & 0 & 0.00 \% & 0.00 \% \\ \text { Total Liptinite } & & \mathbf{3 . 8 6 \%} & \mathbf{4 . 0 0 \%} \\ \text { Detroinertinite } & 104 & 19.12 \% & 19.81 \% \\ \text { Fusinite } & 23 & 4.23 \% & 4.38 \% \\ \text { Semifusinite } & 33 & 6.07 \% & 6.29 \% \\ \text { Macrinite } & 15 & 2.76 \% & 2.86 \% \\ \text { Funginite } & 0 & 0.00 \% & 0.00 \% \\ \text { Total Inertinite } & & \mathbf{3 2 . 1 7 \%} & \mathbf{3 3 . 3 3 \%} \\ \text { clay/other } & 17 & 3.13 \% & \\ \text { quartz } & 2 & 0.37 \% & \\ \text { carbonate } & 0 & 0.00 \% & \mathbf{1 0 0 . 0 0 \%} \\ \text { pyrite } & 0 & 0.00 \% & \\ \text { Total Minerals } & & \mathbf{3 . 4 9 \%} & \\ \text { Total Sum } & 544 & \mathbf{1 0 0 . 0 0 \%} & \end{array}$


Table 9. Vitrinite reflectance data for CZ Fee A No. 114 CBM well, sample 4 (2,679.0-2,681.0 ft).

$\begin{array}{llllll}\text { Readings } & & & & \\ 0.26 & 0.28 & 0.28 & 0.29 & 0.30 & 0.31 \\ 0.33 & 0.34 & 0.34 & 0.34 & 0.35 & 0.35 \\ 0.37 & 0.38 & 0.38 & 0.38 & 0.38 & 0.38 \\ 0.40 & 0.41 & 0.41 & 0.42 & 0.42 & 0.42 \\ 0.44 & 0.45 & 0.45 & 0.47 & 0.67 & 0.68 \\ 0.32 & 0.32 & 0.32 & 0.33 & 0.33 & 0.33 \\ 0.35 & 0.35 & 0.35 & 0.35 & 0.36 & 0.36 \\ 0.38 & 0.38 & 0.39 & 0.39 & 0.39 & 0.40 \\ 0.42 & 0.43 & 0.43 & 0.43 & 0.43 & 0.43 \\ & & & & & \\ \text { Total } & \text { Minimum } & \text { Maximum } & \text { Mean } & \text { Standard } & \\ \text { Readings } & \text { Reflectance } & \text { Reflectance } & \text { Reflectance } & \text { Deviation } & \text { Coal Rank } \\ & & & & & \text { Subbituminous } \\ 54 & 0.26 \% & 0.68 \% & 0.38 \% & 0.075 \% & \text { C }\end{array}$


Table 10. Vitrinite reflectance data for CZ Fee A No. 114 CBM well, sample $7(2,731.0-2,732.0 \mathrm{ft})$.

$\begin{array}{llllll}\text { Readings } & & & & \\ 0.26 & 0.27 & 0.29 & 0.31 & 0.32 & 0.33 \\ 0.35 & 0.36 & 0.36 & 0.36 & 0.37 & 0.37 \\ 0.39 & 0.39 & 0.39 & 0.39 & 0.39 & 0.39 \\ 0.41 & 0.42 & 0.42 & 0.42 & 0.42 & 0.42 \\ 0.46 & 0.46 & 0.46 & 0.48 & 0.49 & 0.55 \\ 0.33 & 0.33 & 0.33 & 0.34 & 0.34 & 0.35 \\ 0.38 & 0.38 & 0.38 & 0.38 & 0.38 & 0.39 \\ 0.39 & 0.40 & 0.40 & 0.40 & 0.40 & 0.41 \\ 0.43 & 0.43 & 0.44 & 0.44 & 0.44 & 0.46 \\ & & & & & \\ \text { Total } & \text { Minimum } & \text { Maximum } & \text { Mean } & \text { Standard } & \\ \text { Readings } & \text { Reflectance } & \text { Reflectance } & \text { Reflectance } & \text { Deviation } & \text { Coal Rank } \\ & & & & & \text { Subbituminous } \\ 54 & 0.26 \% & 0.55 \% & 0.39 \% & 0.05 \% & \text { C }\end{array}$


Table 11. Vitrinite reflectance data for CZ Fee A No. 114 CBM well, sample 11 (2,938.0-2,939.0 ft).

$\begin{array}{llllll}\text { Readings } & & & & & \\ 0.26 & 0.27 & 0.28 & 0.28 & 0.29 & 0.29 \\ 0.30 & 0.31 & 0.31 & 0.31 & 0.31 & 0.32 \\ 0.33 & 0.33 & 0.33 & 0.34 & 0.34 & 0.34 \\ 0.36 & 0.37 & 0.37 & 0.37 & 0.38 & 0.38 \\ 0.43 & 0.45 & 0.47 & 0.50 & 0.30 & 0.30 \\ 0.29 & 0.29 & 0.29 & 0.29 & 0.33 & 0.33 \\ 0.32 & 0.32 & 0.33 & 0.33 & 0.36 & 0.36 \\ 0.35 & 0.35 & 0.35 & 0.36 & 0.41 & 0.41 \\ 0.39 & 0.39 & 0.40 & 0.40 & & \end{array}$

$\begin{array}{llllll}\text { Total } & \text { Minimum } & \text { Maximum } & \text { Mean } & \text { Standard } & \text { Coal } \\ \text { Readings } & \text { Reflectance } & \text { Reflectance } & \text { Reflectance } & \text { Deviation } & \text { Rank } \\ 52 & 0.26 \% & 0.5 \% & 0.34 \% & 0.05 \% & \text { Lignite }\end{array}$


Table 12. Methane adsorption isotherm analytical data (as-analyzed basis) for CZ Fee $A$ No. 114 CBM well, sample 4 (2,679.0-2,681.0 ft). Abbreviations: $g$ = grams; \% = percent; ${ }^{\circ} \mathrm{F}=$ degrees Fahrenheit; ${ }^{\circ} \mathrm{C}=$ degrees Celsius; $p s i=$ pounds per square inch; $\mathrm{MPa}=$ Mega Pascal; scf/ton = standard cubic feet per ton; scc/gm = standard cubic centimeters per gram; $V=$ volume; $P=$ pressure.

Methane Adsorption Isotherm Vintage Petroleum

Sample: 4

As-Analyzed Basis
Well: CZ Fee A No. 114

Depth: 2,679.0'-81.0'

\begin{tabular}{|ll|}
\hline Sample Weight $=131.36 \mathrm{~g}$ & Ash Content $=33.30 \%$ \\
Particle Size $=<20 \mathrm{Mesh}$ & Equilibrium Moisture Content $=18.55 \%$ \\
Temperature $=110.0^{\circ} \mathrm{F}\left(43.3^{\circ} \mathrm{C}\right)$ & \\
\hline
\end{tabular}

\section{Methane Adsorption}

\begin{tabular}{|cc|cc|}
\hline $\begin{array}{c}\text { Pressure } \\
(\mathrm{psi})\end{array}$ & $(\mathrm{MPa})$ & \multicolumn{2}{c|}{ Gas Content (Raw Basis) } \\
\\
52 & 0.36 & $(\mathrm{scf} /$ ton $)$ & 0.24 \\
117 & 0.81 & 1.7 & 0.49 \\
215 & 1.48 & 26.8 & 0.84 \\
354 & 2.44 & 40.7 & 1.27 \\
544 & 3.75 & 55.2 & 1.72 \\
833 & 5.74 & 71.4 & 2.23 \\
1,232 & 8.49 & 88.1 & 2.75 \\
1,655 & 11.41 & 98.9 & 3.09 \\
2,151 & 14.83 & 109.2 & 3.41 \\
\hline
\end{tabular}

\section{Langmuir Coefficients}

$V=164.4{ }^{*} P /(P+1,085.0)$

\begin{tabular}{|c|c|c|c|}
\hline \multicolumn{2}{|c|}{$\underline{P L}$} & \multicolumn{2}{|c|}{$\underline{\mathrm{VL} \text { (Raw Basis) }}$} \\
\hline (psi) & $(\mathrm{MPa})$ & (scf/ton) & (scc/gm) \\
\hline $1,085.0$ & 7.48 & 164.4 & 5.1 \\
\hline
\end{tabular}


Table 13. Methane adsorption isotherm analytical data (dry basis) for CZ Fee A No. 114 CBM well, sample $4(2,679 \cdot 0-2,681.0 \mathrm{ft})$. Abbreviations: $\mathrm{g}=$ grams; $\%=$ percent; ${ }^{\circ} \mathrm{F}=$ degrees Fahrenheit; ${ }^{\circ} \mathrm{C}=$ degrees Celsius; $p s i=$ pounds per square inch; $\mathrm{MPa}=\mathrm{Mega}$ Pascal; scf/ton = standard cubic feet per ton; scc/gm = standard cubic centimeters per gram; $\mathrm{V}=$ volume; $\mathrm{P}=$ pressure .

Methane Adsorption Isotherm

Vintage Petroleum

Well: CZ Fee A No. 114

Sample: 4

Depth: 2,679.0'-81.0'

Dry Basis

\begin{tabular}{|ll}
\hline Sample Weight $=131.36 \mathrm{~g}$ & Ash Content $=33.30 \%$ \\
Particle Size $=<20 \mathrm{Mesh}$ & Equilibrium Moisture Content $=18.55 \%$ \\
Temperature $=110.0^{\circ} \mathrm{F}\left(43.3^{\circ} \mathrm{C}\right)$ & \\
\hline
\end{tabular}

Methane Adsorption

\begin{tabular}{|cc|cc|}
\hline $\begin{array}{c}\text { Pressure } \\
(\mathrm{psi})\end{array}$ & $(\mathrm{MPa})$ & \multicolumn{2}{c|}{ Gas Content (Dry Basis) } \\
(scf/ton) & (scc/gm) \\
\hline 52 & 0.36 & 9.4 & 0.29 \\
117 & 0.81 & 19.4 & 0.61 \\
215 & 1.48 & 33.0 & 1.03 \\
354 & 2.44 & 50.0 & 1.56 \\
544 & 3.75 & 67.8 & 2.12 \\
833 & 5.74 & 87.7 & 2.74 \\
1,232 & 8.49 & 108.2 & 3.38 \\
1,655 & 11.41 & 121.4 & 3.79 \\
2,151 & 14.83 & 134.1 & 4.19 \\
\hline
\end{tabular}

Langmuir Coefficients $\quad V=201.9 * P /(P+1,085.0)$

\begin{tabular}{|c|c|c|c|}
\hline \multicolumn{2}{|c|}{$\underline{P L}$} & \multicolumn{2}{|c|}{$\underline{\text { VL (Dry Basis) }}$} \\
\hline (psi) & (MPa) & (scf/ton) & (scc/gm) \\
\hline $1,085.0$ & 7.48 & 201.9 & 6.3 \\
\hline
\end{tabular}


Table 14. Methane adsorption isotherm analytical data (dry, ash-free basis) for CZ Fee $A$ No. 114 CBM well, sample 4 (2,679.0-2,681.0 ft). Abbreviations: $g$ = grams; $\%$ = percent; ${ }^{\circ} \mathrm{F}$ $=$ degrees Fahrenheit; ${ }^{\circ} \mathrm{C}=$ degrees Celsius; $p s i=$ pounds per square inch; $\mathrm{MPa}=\mathrm{Mega}$ Pascal; scf/ton = standard cubic feet per ton; scc/gm = standard cubic centimeters per gram; $\mathrm{V}=$ volume; $\mathrm{P}=$ pressure .

Methane Adsorption Isotherm

Vintage Petroleum

Sample: 4

Dry, Ash-Free Basis
Well: CZ Fee A No. 114

Depth: 2,679.0'-81.0'
Sample Weight $=131.36 \mathrm{~g}$

Particle Size $=<20$ Mesh

Temperature $=110.0^{\circ} \mathrm{F}\left(43.3^{\circ} \mathrm{C}\right)$
Ash Content $=33.30 \%$

Equilibrium Moisture Content $=18.55 \%$

\section{Methane Adsorption}

\begin{tabular}{|cc|cc|}
\hline $\begin{array}{c}\text { Pressure } \\
(\mathrm{psi})\end{array}$ & $(\mathrm{MPa})$ & \multicolumn{2}{c|}{ Gas Content (Dry, Ash-Free Basis) } \\
\hline 52 & 0.36 & $(\mathrm{scf} /$ ton $)$ & 0.50 \\
117 & 0.81 & 15.9 & 1.02 \\
215 & 1.48 & 32.8 & 1.74 \\
354 & 2.44 & 55.8 & 2.64 \\
544 & 3.75 & 84.6 & 3.58 \\
833 & 5.74 & 114.7 & 4.63 \\
1,232 & 8.49 & 148.3 & 5.71 \\
1,655 & 11.41 & 183.0 & 6.41 \\
2,151 & 14.83 & 205.3 & 7.08 \\
\hline
\end{tabular}

Langmuir Coefficients $\quad V=341.5 * P /(P+1,085.0)$

\begin{tabular}{|c|c|c|c|}
\hline \multicolumn{2}{|c|}{$\underline{\mathrm{PL}}$} & \multicolumn{2}{|c|}{$\underline{\text { VL (Dry, Ash-Free Basis) }}$} \\
\hline (psi) & $(\mathrm{MPa})$ & (scf/ton) & (scc/gm) \\
\hline $1,085.0$ & 7.48 & 341.5 & 10.6 \\
\hline
\end{tabular}


Table 15. Methane adsorption isotherm analytical data (as-analyzed basis) for CZ Fee $\mathrm{A}$ No. 114 CBM well, sample 7 (2,731.0-2,732.0 ft). Abbreviations: $g$ = grams; $\%=$ percent; ${ }^{\circ} \mathrm{F}=$ degrees Fahrenheit; ${ }^{\circ} \mathrm{C}=$ degrees Celsius; $p s i=$ pounds per square inch; $\mathrm{MPa}=$ Mega Pascal; scf/ton = standard cubic feet per ton; scc/gm = standard cubic centimeters per gram; $V=$ volume; $P=$ pressure.

Methane Adsorption Isotherm Vintage Petroleum

Sample: 7

As-Analyzed Basis
Well: CZ Fee A No. 114

Depth: 2,731.0'-32.0'

\begin{tabular}{|ll|}
\hline Sample Weight $=134.03 \mathrm{~g}$ & Ash Content $=46.99 \%$ \\
Particle Size $=<20 \mathrm{Mesh}$ & Equilibrium Moisture Content $=16.20 \%$ \\
Temperature $=110.0^{\circ} \mathrm{F}\left(43.3^{\circ} \mathrm{C}\right)$ & \\
\hline
\end{tabular}

\section{Methane Adsorption}

\begin{tabular}{|cc|cc|}
\hline $\begin{array}{c}\text { Pressure } \\
(\mathrm{psi})\end{array}$ & $(\mathrm{MPa})$ & \multicolumn{2}{c|}{ Gas Content (Raw Basis) } \\
\\
62 & 0.43 & $(\mathrm{scf} /$ ton $)$ & 0.18 \\
115 & 0.79 & 5.7 & 0.33 \\
212 & 1.46 & 10.6 & 0.58 \\
359 & 2.48 & 18.5 & 0.89 \\
536 & 3.70 & 28.4 & 1.20 \\
821 & 5.66 & 38.5 & 1.54 \\
1,211 & 8.35 & 49.4 & 1.92 \\
1,630 & 11.24 & 61.6 & 2.20 \\
2,130 & 14.69 & 70.4 & 2.42 \\
\hline
\end{tabular}

\section{Langmuir Coefficients}

$V=122.0$ * $P /(P+1,202.5)$

\begin{tabular}{|c|c|c|c|}
\hline \multicolumn{2}{|c|}{$\underline{P L}$} & \multicolumn{2}{|c|}{$\underline{\mathrm{VL}}$ (Raw Basis) } \\
\hline (psi) & (MPa) & (scf/ton) & (scc/gm) \\
\hline $1,202.5$ & 8.29 & 122.0 & 3.8 \\
\hline
\end{tabular}


Table 16. Methane adsorption isotherm analytical data (dry basis) for CZ Fee A No. 114 CBM well, sample $7(2,731.0-2,732.0 \mathrm{ft})$. Abbreviations: $\mathrm{g}=$ grams; $\%=$ percent; ${ }^{\circ} \mathrm{F}=$ degrees Fahrenheit; ${ }^{\circ} \mathrm{C}=$ degrees Celsius; $p s i=$ pounds per square inch; $\mathrm{MPa}=\mathrm{Mega}$ Pascal; scf/ton = standard cubic feet per ton; scc/gm = standard cubic centimeters per gram; $V$ = volume; $P=$ pressure.

Methane Adsorption Isotherm Vintage Petroleum

Sample: 7

Dry Basis
Well: CZ Fee A No. 114

Depth: 2,731.0'-32.0'

\begin{tabular}{|ll|}
\hline Sample Weight $=134.03 \mathrm{~g}$ & Ash Content $=46.99 \%$ \\
Particle Size $=<20$ Mesh & Equilibrium Moisture Content $=16.20 \%$ \\
Temperature $=110.0^{\circ} \mathrm{F}\left(43.3^{\circ} \mathrm{C}\right)$ & \\
\hline
\end{tabular}

\section{Methane Adsorption}

\begin{tabular}{|c|c|c|c|}
\hline \multicolumn{2}{|c|}{ Pressure } & \multicolumn{2}{|c|}{ Gas Content (Dry Basis) } \\
\hline (psi) & (MPa) & (scf/ton) & (scc/gm) \\
\hline 62 & 0.43 & 6.8 & 0.21 \\
\hline 115 & 0.79 & 12.6 & 0.39 \\
\hline 212 & 1.46 & 22.1 & 0.69 \\
\hline 359 & 2.48 & 33.9 & 1.06 \\
\hline 536 & 3.70 & 46.0 & 1.44 \\
\hline 821 & 5.66 & 58.9 & 1.84 \\
\hline 1,211 & 8.35 & 73.5 & 2.29 \\
\hline 1,630 & 11.24 & 84.0 & 2.62 \\
\hline 2,130 & 14.69 & 92.4 & 2.88 \\
\hline
\end{tabular}

\section{Langmuir Coefficients}

$V=145.6$ * $P /(P+1,202.5)$

\begin{tabular}{|c|c|c|c|}
\hline \multicolumn{2}{|c|}{$\underline{P L}$} & \multicolumn{2}{|c|}{$\underline{\text { VL (Dry Basis) }}$} \\
\hline (psi) & (MPa) & (scf/ton) & (scc/gm) \\
\hline $1,202.5$ & 8.29 & 145.6 & 4.5 \\
\hline
\end{tabular}


Table 17. Methane adsorption isotherm analytical data (dry, ash-free basis) for CZ Fee A No. 114 CBM well, sample 7 (2,731.0-2,732.0 ft). Abbreviations: $g=$ grams; $\%=$ percent; ${ }^{\circ} \mathrm{F}=$ degrees Fahrenheit; ${ }^{\circ} \mathrm{C}=$ degrees Celsius; $p s i=$ pounds per square inch; $\mathrm{MPa}=$ Mega Pascal; scf/ton = standard cubic feet per ton; scc/gm = standard cubic centimeters per gram; $V$ = volume; $\mathrm{P}=$ pressure.

\section{Methane Adsorption Isotherm}

Vintage Petroleum

Sample: 7

Dry, Ash-Free Basis
Well: CZ Fee A No. 114

Depth: 2,731.0'-32.0'
Sample Weight $=134.03 \mathrm{~g}$

Particle Size $=<20$ Mesh

Temperature $=110.0^{\circ} \mathrm{F}\left(43.3^{\circ} \mathrm{C}\right)$
Ash Content $=46.99 \%$

Equilibrium Moisture Content $=16.20 \%$

\section{Methane Adsorption}

\begin{tabular}{|cc|cc|}
\hline $\begin{array}{c}\text { Pressure } \\
(\mathrm{psi})\end{array}$ & $(\mathrm{MPa})$ & \multicolumn{2}{c|}{ Gas Content (Dry, Ash-Free Basis) } \\
62 & 0.43 & $($ scf/ton $)$ & 0.48 \\
115 & 0.79 & 15.5 & 0.90 \\
212 & 1.46 & 28.7 & 1.57 \\
359 & 2.48 & 50.3 & 2.41 \\
536 & 3.70 & 77.2 & 3.27 \\
821 & 5.66 & 104.7 & 4.19 \\
1,211 & 8.35 & 134.1 & 5.22 \\
1,630 & 11.24 & 167.3 & 5.97 \\
2,130 & 14.69 & 191.2 & 6.56 \\
\hline
\end{tabular}

Langmuir Coefficients $\quad V=331.4$ * $P /(P+1,202.5)$

\begin{tabular}{|c|c|c|c|}
\hline \multicolumn{2}{|c|}{$\underline{\mathrm{PL}}$} & \multicolumn{2}{|c|}{$\underline{\text { VL (Dry, Ash-Free Basis) }}$} \\
\hline (psi) & $(\mathrm{MPa})$ & (scf/ton) & (scc/gm) \\
\hline $1,202.5$ & 8.29 & 331.4 & 10.3 \\
\hline
\end{tabular}


Table 18. Methane adsorption isotherm analytical data (as-analyzed basis) for CZ Fee $\mathrm{A}$ No. 114 CBM well, sample 11 (2,938.0-2,939.0 ft). Abbreviations: $g=$ grams; $\%=$ percent; ${ }^{\circ} \mathrm{F}=$ degrees Fahrenheit; ${ }^{\circ} \mathrm{C}=$ degrees Celsius; $p s i=$ pounds per square inch; $\mathrm{MPa}=$ Mega Pascal; scf/ton = standard cubic feet per ton; scc/gm = standard cubic centimeters per gram; $V=$ volume; $P=$ pressure.

Methane Adsorption Isotherm Vintage Petroleum

Sample: 11

As-Analyzed Basis
Well: CZ Fee A No. 114

Depth: 2,938.0'-39.0'

\begin{tabular}{|ll|}
\hline Sample Weight $=87.03 \mathrm{~g}$ & Ash Content $=6.46 \%$ \\
Particle Size $=<20 \mathrm{Mesh}$ & Equilibrium Moisture Content $=23.42 \%$ \\
Temperature $=110.0^{\circ} \mathrm{F}\left(43.3^{\circ} \mathrm{C}\right)$ & \\
\hline
\end{tabular}

\begin{tabular}{|c|c|c|c|}
\hline \multicolumn{4}{|c|}{ Methane Adsorption } \\
\hline \multicolumn{2}{|c|}{ Pressure } & \multicolumn{2}{|c|}{$\underline{\text { Gas Content (Raw Basis) }}$} \\
\hline (psi) & $(\mathrm{MPa})$ & (scf/ton) & (scc/gm) \\
\hline 58 & 0.40 & 10.8 & 0.34 \\
\hline 110 & 0.76 & 21.1 & 0.66 \\
\hline 202 & 1.39 & 36.9 & 1.15 \\
\hline 343 & 2.36 & 57.5 & 1.79 \\
\hline 517 & 3.56 & 71.8 & 2.24 \\
\hline 792 & 5.46 & 96.5 & 3.01 \\
\hline 1,173 & 8.09 & 118.9 & 3.71 \\
\hline 1,581 & 10.90 & 137.2 & 4.28 \\
\hline 2,070 & 14.27 & 150.4 & 4.69 \\
\hline \multicolumn{4}{|c|}{ Langmuir Coefficients } \\
\hline \multicolumn{2}{|c|}{$\underline{\mathrm{PL}}$} & \multicolumn{2}{|c|}{$\underline{\text { VL (Raw Basis) }}$} \\
\hline (psi) & $(\mathrm{MPa})$ & (scf/ton) & (scc/gm) \\
\hline $1,109.1$ & 7.65 & 231.9 & 7.2 \\
\hline
\end{tabular}


Table 19. Methane adsorption isotherm analytical data (dry basis) for CZ Fee A No. 114 CBM well, sample $11(2,938.0-2,939.0 \mathrm{ft})$. Abbreviations: $\mathrm{g}=$ grams; $\%=$ percent; ${ }^{\circ} \mathrm{F}=$ degrees Fahrenheit; ${ }^{\circ} \mathrm{C}=$ degrees Celsius; $p s i=$ pounds per square inch; $\mathrm{MPa}=\mathrm{Mega}$ Pascal; scf/ton = standard cubic feet per ton; scc/gm = standard cubic centimeters per gram; $V=$ volume; $P$ = pressure.

\section{Methane Adsorption Isotherm}

Vintage Petroleum

Sample: 11

Dry Basis
Well: CZ Fee A No. 114

Depth: 2,938.0'-39.0'
Sample Weight $=87.03 \mathrm{~g}$

Particle Size $=<20$ Mesh

Temperature $=110.0^{\circ} \mathrm{F}\left(43.3^{\circ} \mathrm{C}\right)$
Ash Content $=6.46 \%$

Equilibrium Moisture Content $=23.42 \%$

\begin{tabular}{|cc|cc|}
\hline Methane Adsorption & \multicolumn{3}{l|}{} \\
\hline Pressure & & Gas Content (Dry Basis) & \\
$(\mathrm{psi})$ & $(\mathrm{MPa})$ & $\frac{}{\text { (scf/ton })}$ & $(\mathrm{scc} / \mathrm{gm})$ \\
\hline 58 & 0.40 & 14.1 & 0.44 \\
110 & 0.76 & 27.5 & 0.86 \\
202 & 1.39 & 48.2 & 1.50 \\
343 & 2.36 & 75.1 & 2.34 \\
517 & 3.56 & 93.8 & 2.93 \\
792 & 5.46 & 126.0 & 3.93 \\
1,173 & 8.09 & 155.3 & 4.85 \\
1,581 & 10.90 & 179.1 & 5.59 \\
2,070 & 14.27 & 196.4 & 6.13 \\
\hline
\end{tabular}

\section{Langmuir Coefficients $\quad V=302.8$ * $P /(P+1,109.1)$}

\begin{tabular}{|c|c|c|c|}
\hline \multicolumn{2}{|c|}{$\underline{P L}$} & \multicolumn{2}{|c|}{$\underline{\text { VL (Dry Basis) }}$} \\
\hline (psi) & (MPa) & (scf/ton) & (scc/gm) \\
\hline $1,109.1$ & 7.65 & 302.8 & 9.4 \\
\hline
\end{tabular}


Table 20. Methane adsorption isotherm analytical data (dry, ash-free basis) for CZ Fee A No. 114 CBM well, sample 11 (2,938.0-2,939.0 ft). Abbreviations: $g=$ grams; $\%=$ percent; ${ }^{\circ} \mathrm{F}=$ degrees Fahrenheit; ${ }^{\circ} \mathrm{C}=$ degrees Celsius; $p s i=$ pounds per square inch; $\mathrm{MPa}=$ Mega Pascal; scf/ton = standard cubic feet per ton; scc/gm = standard cubic centimeters per gram; $V=$ volume; $P=$ pressure .

Methane Adsorption Isotherm

Vintage Petroleum

Sample: 11

Dry, Ash-Free Basis
Well: CZ Fee A No. 114

Depth: 2,938.0'-39.0'
Sample Weight $=87.03 \mathrm{~g}$

Particle Size $=<20$ Mesh

Temperature $=110.0^{\circ} \mathrm{F}\left(43.3^{\circ} \mathrm{C}\right)$
Ash Content $=6.46 \%$

Equilibrium Moisture Content $=23.42 \%$

\section{Methane Adsorption}

\begin{tabular}{|cc|cc|}
\hline $\begin{array}{c}\text { Pressure } \\
(\mathrm{psi})\end{array}$ & $(\mathrm{MPa})$ & \multicolumn{2}{c|}{ Gas Content (Dry, Ash-Free Basis) } \\
\hline 58 & 0.40 & $(\mathrm{scf} / \mathrm{ton})$ & 0.48 \\
110 & 0.76 & 15.4 & 0.94 \\
202 & 1.39 & 30.0 & 1.64 \\
343 & 2.36 & 52.6 & 2.56 \\
517 & 3.56 & 82.0 & 3.20 \\
792 & 5.46 & 102.4 & 4.30 \\
1,173 & 8.09 & 137.6 & 5.29 \\
1,581 & 10.90 & 169.6 & 6.11 \\
2,070 & 14.27 & 195.6 & 6.70 \\
\hline
\end{tabular}

Langmuir Coefficients $\quad V=330.7 * P /(P+1,109.1)$

\begin{tabular}{|cc|cc|}
\hline$\underline{P L}$ & \multicolumn{2}{|c|}{ VL (Dry, Ash-Free Basis) } & \\
$(\mathrm{psi})$ & $(\mathrm{MPa})$ & $(\mathrm{scf} / \mathrm{ton})$ & 10.3 \\
\hline $1,109.1$ & 7.65 & 330.7 & \\
\hline
\end{tabular}


Table 21. Produced water data for CZ Fee A No. 114 CBM well, February 2004. Abbreviations: TDS $=$ total dissolved solids; $\mathrm{mg} / \mathrm{l}=$ milligrams per liter; $\mathrm{g} / \mathrm{cm}^{3}=$ grams per cubic centimeter; ${ }^{\circ} \mathrm{F}=$ degrees Fahrenheit; $\mathrm{meq} / \mathrm{l}=$ milliequivalents per liter.

\begin{tabular}{|c|c|c|}
\hline \multicolumn{3}{|l|}{ Summary } \\
\hline Sampling Date & $\begin{array}{c}25-F e b- \\
04\end{array}$ & \\
\hline Analysis Date & $\begin{array}{l}\text { 4-Mar- } \\
04\end{array}$ & \\
\hline TDS (mg/l) & $82,819.6$ & \\
\hline Density $\left(\mathrm{g} / \mathrm{cm}^{3}\right)$ & 1.061 & \\
\hline $\begin{array}{l}\text { Anion/Cation } \\
\text { Ratio }\end{array}$ & 1.00 & \\
\hline Anions $\left(75^{\circ} \mathrm{F}\right)$ & $\mathrm{mg} / \mathrm{l}$ & $\mathrm{meq} / \mathrm{l}$ \\
\hline Chloride & 50,100 & 1,413 \\
\hline Bicarbonate & 226 & 3.70 \\
\hline Sulfate & 44.8 & 0.93 \\
\hline $\mathrm{pH}$ at time of sar & pling & 5.85 \\
\hline $\mathrm{pH}$ at time of an & ysis & 5.85 \\
\hline $\mathrm{pH}$ used in calcu & ations & 5.85 \\
\hline Cations $\left(75^{\circ} \mathrm{F}\right)$ & $\mathrm{mg} / \mathrm{l}$ & $\mathrm{meq} / \mathrm{l}$ \\
\hline Sodium & 29,133 & 1,267 \\
\hline Magnesium & 171 & 14.1 \\
\hline Calcium & 2,520 & 126 \\
\hline Barium & 549 & 8.00 \\
\hline Iron & 75.3 & 2.70 \\
\hline Manganese & 0.96 & 0.03 \\
\hline
\end{tabular}


Table 22. Produced water data for CZ Fee A No. 114 CBM well, March 2004. Abbreviations: TDS = total dissolved solids; $\mathrm{mg} / \mathrm{l}=$ milligrams per liter; $\mathrm{g} / \mathrm{cm}^{3}=$ grams per cubic centimeter; ${ }^{\circ} \mathrm{F}=$ degrees Fahrenheit; $\mathrm{meq} / \mathrm{l}=$ milliequivalents per liter.

\begin{tabular}{|c|c|c|}
\hline $\begin{array}{l}\text { Summary } \\
\text { Sampling Date }\end{array}$ & $\begin{array}{c}\text { 25-Mar- } \\
04\end{array}$ & \\
\hline Analysis Date & 6-Apr-04 & \\
\hline $\begin{array}{l}\text { TDS }(\mathrm{mg} / \mathrm{l}) \\
\text { Density }\left(\mathrm{g} / \mathrm{cm}^{3}\right)\end{array}$ & $\begin{array}{c}84,598.4 \\
1.063\end{array}$ & \\
\hline $\begin{array}{l}\text { Anion/Cation } \\
\text { Ratio }\end{array}$ & 1.00 & \\
\hline Anions $\left(75^{\circ} \mathrm{F}\right)$ & $\mathrm{mg} / \mathrm{l}$ & meq/l \\
\hline Chloride & 51,100 & 1,441 \\
\hline Bicarbonate & 372 & 6.10 \\
\hline Sulfate & 0.39 & 0.01 \\
\hline $\mathrm{pH}$ at time of sar & pling & 6.58 \\
\hline $\mathrm{pH}$ at time of an & ysis & 6.58 \\
\hline $\mathrm{pH}$ used in calcu & ations & 6.58 \\
\hline Cations $\left(75^{\circ} \mathrm{F}\right)$ & $\mathrm{mg} / \mathrm{l}$ & meq/l \\
\hline Sodium & 29,696 & 1,292 \\
\hline Magnesium & 97.6 & 8.03 \\
\hline Calcium & 2,800 & 140 \\
\hline Barium & 521 & 7.59 \\
\hline Iron & 10.9 & 0.39 \\
\hline Manganese & 0.34 & 0.01 \\
\hline
\end{tabular}


Table 23. Gas-in-place (GIP) calculations for CZ Fee A No. 114 CBM well.

\begin{tabular}{|c|c|c|c|c|c|c|c|}
\hline \multicolumn{8}{|l|}{ Assumptions: } \\
\hline Drainage & 160 & Acres & & & & & \\
\hline \multicolumn{8}{|c|}{ Core \#1 } \\
\hline Depth (ft) & $\mathrm{C}_{\mathrm{gi}}$ (scf/ton) & $\rho_{c}\left(g / \mathrm{cm}^{3}\right)$ & $f_{a}$ & $f_{m}$ & A (acres) & $h(f t)$ & GIP (Mcf) \\
\hline 2,672 & 135.4 & 1.65 & 0.403 & 0.116 & 160 & 0.5 & 11,690 \\
\hline $2,672.5$ & 135.4 & 1.35 & 0.155 & 0.116 & 160 & 0.5 & 14,481 \\
\hline 2,673 & 135.4 & 1.3 & 0.114 & 0.116 & 160 & 0.5 & 14,734 \\
\hline $2,673.5$ & 135.4 & 1.25 & 0.073 & 0.116 & 160 & 0.5 & 14,926 \\
\hline 2,674 & 135.4 & 1.26 & 0.081 & 0.116 & 160 & 0.5 & 14,892 \\
\hline $2,674.5$ & 135.4 & 1.27 & 0.089 & 0.116 & 160 & 0.5 & 14,856 \\
\hline 2,675 & 135.4 & 1.3 & 0.114 & 0.116 & 160 & 0.5 & 14,734 \\
\hline $2,675.5$ & 135.4 & 1.27 & 0.089 & 0.116 & 160 & 0.5 & 14,856 \\
\hline 2,676 & 135.4 & 1.45 & 0.238 & 0.116 & 160 & 0.5 & 13,793 \\
\hline 2,679 & 135.4 & 1.7 & 0.444 & 0.116 & 160 & 0.5 & 11,012 \\
\hline $2,679.5$ & 135.4 & 1.4 & 0.197 & 0.116 & 160 & 0.5 & 14,167 \\
\hline 2,680 & 135.4 & 1.33 & 0.139 & 0.116 & 160 & 0.5 & 14,589 \\
\hline $2,680.5$ & 135.4 & 1.32 & 0.131 & 0.116 & 160 & 0.5 & 14,640 \\
\hline 2,681 & 135.4 & 1.41 & 0.205 & 0.116 & 160 & 0.5 & 14,097 \\
\hline $2,681.5$ & 135.4 & 1.7 & 0.444 & 0.116 & 160 & 0.5 & 11,012 \\
\hline Total core 1 & & & & & & & 208,479 \\
\hline \multicolumn{8}{|c|}{ Core \#2 } \\
\hline Depth (ft) & $\mathrm{C}_{\mathrm{gi}}$ (scf/ton) & $\rho_{\mathrm{c}}\left(\mathrm{g} / \mathrm{cm}^{3}\right)$ & $\boldsymbol{f}_{\boldsymbol{a}}$ & $\boldsymbol{f}_{m}$ & A (acres) & $h(f t)$ & GIP (Mcf) \\
\hline 2,730 & 171.6 & 1.55 & 0.320 & 0.148 & 160 & 0.5 & 15,376 \\
\hline $2,730.5$ & 171.6 & 1.57 & 0.337 & 0.148 & 160 & 0.5 & 15,092 \\
\hline 2,731 & 171.6 & 1.41 & 0.205 & 0.148 & 160 & 0.5 & 17,025 \\
\hline $2,731.5$ & 171.6 & 1.29 & 0.106 & 0.148 & 160 & 0.5 & 17,958 \\
\hline 2,732 & 171.6 & 1.25 & 0.073 & 0.148 & 160 & 0.5 & 18,170 \\
\hline $2,732.5$ & 171.6 & 1.3 & 0.114 & 0.148 & 160 & 0.5 & 17,897 \\
\hline 2,733 & 171.6 & 1.32 & 0.131 & 0.148 & 160 & 0.5 & 17,766 \\
\hline $2,733.5$ & 171.6 & 1.45 & 0.238 & 0.148 & 160 & 0.5 & 16,615 \\
\hline 2,734 & 171.6 & 1.9 & 0.609 & 0.148 & 160 & 0.5 & 8,617 \\
\hline Total core 2 & & & & & & & 144,515 \\
\hline \multicolumn{8}{|c|}{ Core \#3 } \\
\hline Depth (ft) & $\mathrm{C}_{\mathrm{gi}}$ (scf/ton) & $\rho_{c}\left(g / \mathrm{cm}^{3}\right)$ & $f_{a}$ & $f_{m}$ & A (acres) & $h(f t)$ & GIP (Mcf) \\
\hline 2,938 & 186.4 & 1.78 & 0.510 & 0.1723 & 160 & 0.5 & 14,462 \\
\hline $2,938.5$ & 186.4 & 1.38 & 0.180 & 0.1723 & 160 & 0.5 & 18,112 \\
\hline 2,939 & 186.4 & 1.3 & 0.114 & 0.1723 & 160 & 0.5 & 18,800 \\
\hline $2,939.5$ & 186.4 & 1.35 & 0.155 & 0.1723 & 160 & 0.5 & 18,395 \\
\hline 2,940 & 186.4 & 1.3 & 0.114 & 0.1723 & 160 & 0.5 & 18,800 \\
\hline $2,940.5$ & 186.4 & 1.3 & 0.114 & 0.1723 & 160 & 0.5 & 18,800 \\
\hline 2,941 & 186.4 & 1.3 & 0.114 & 0.1723 & 160 & 0.5 & 18,800 \\
\hline $2,941.5$ & 186.4 & 1.3 & 0.114 & 0.1723 & 160 & 0.5 & 18,800 \\
\hline 2,942 & 186.4 & 1.3 & 0.114 & 0.1723 & 160 & 0.5 & 18,800 \\
\hline $2,942.5$ & 186.4 & 1.3 & 0.114 & 0.1723 & 160 & 0.5 & 18,800 \\
\hline 2,943 & 186.4 & 1.6 & 0.362 & 0.1723 & 160 & 0.5 & 15,116 \\
\hline Total core 3 & & & & & & & 194,686 \\
\hline GIP Total & ns & & & & & & $\begin{array}{l}547,680 \\
\text { Icf }\end{array}$ \\
\hline
\end{tabular}

Explanation: Depth $=$ top of $0.5 \mathrm{ft}$ sample interval, $\mathrm{C}_{\mathrm{gi}}=$ average measured desorbed gas content for entire cored interval (see Table 2), dry ash-free, $\rho_{\mathrm{c}}=$ bulk density, $\mathrm{f}_{\mathrm{a}}=$ ash fraction of $0.5 \mathrm{ft}$ sample interval (calculated from bulk density), $\mathrm{f}_{\mathrm{m}}=$ moisture fraction of $0.5 \mathrm{ft}$ interval, $A=$ area, $h=$ height, $G I P=$ gas in place $=A \cdot h \cdot\left[1.359 \cdot C_{g i} \cdot \rho_{c} \cdot\left(1-f_{a}-f_{m}\right)\right]$. Abbreviations: scf/ton $=$ standard cubic feet per ton; $g / \mathrm{cm}^{3}$ $=$ grams per cubic centimeter; $\mathrm{ft}=$ feet; $\mathrm{Mcf}=$ thousand cubic feet 


\section{APPENDIX: Permission to publish coalbed methane analytical data from operator}

\author{
"Troy Richard" \\ <trichard@hilcorp.com> \\ 06/26/2007 08:42 AM
}

\author{
To "Paul C Hackley" <phackley@usgs.gov> \\ CC \\ Subjec RE: Release of Colgrade CZ "A" \#114 cbm data
}

Mr. Hackley,

On May 9, 2007, I obtain approval from Hilcorp's Executive Vice President - Exploitation. You have Hilcorp's permission to publish the information as written.

Troy E. Richard, CPL

Senior Landman

Hilcorp Energy Company, General Partner

For Hilcorp Energy I, L.P.

(713) 289-2651 Phone

(713) 209-2420 Fax 